\title{
Review Article \\ Experimental Results on Chiral Magnetic and Vortical Effects
}

\author{
Gang Wang and Liwen Wen \\ Department of Physics and Astronomy, University of California, Los Angeles, CA 90095, USA \\ Correspondence should be addressed to Gang Wang; gwang@physics.ucla.edu
}

Received 11 September 2016; Accepted 1 December 2016; Published 12 January 2017

Academic Editor: Declan Keane

Copyright (C) 2017 G. Wang and L. Wen. This is an open access article distributed under the Creative Commons Attribution License, which permits unrestricted use, distribution, and reproduction in any medium, provided the original work is properly cited. The publication of this article was funded by SCOAP ${ }^{3}$.

\begin{abstract}
Various novel transport phenomena in chiral systems result from the interplay of quantum anomalies with magnetic field and vorticity in high-energy heavy-ion collisions and could survive the expansion of the fireball and be detected in experiments. Among them are the chiral magnetic effect, the chiral vortical effect, and the chiral magnetic wave, the experimental searches for which have aroused extensive interest. The goal of this review is to describe the current status of experimental studies at Relativistic Heavy-Ion Collider at BNL and the Large Hadron Collider at CERN and to outline the future work in experiment needed to eliminate the existing uncertainties in the interpretation of the data.
\end{abstract}

\section{Introduction}

High-energy heavy-ion collisions can produce a hot, dense, and deconfined nuclear medium, which is dubbed the quarkgluon plasma (QGP). The thermodynamic states of a QGP can be specified by the axial chemical potential $\mu_{5}$, besides the temperature $T$ and the vector chemical potential $\mu . \mu_{5}$ characterizes the imbalance of right-handed and left-handed fermions in a system, and a chiral system bears a nonzero $\mu_{5}$. Chiral domains may be created locally in heavy-ion collisions through various mechanisms on an event-by-event basis (e.g., topological fluctuations in the gluonic sector, glasma flux tubes, or fluctuations in the quark sector) [1-6]. In a noncentral collision, a strong magnetic field $\left(B \sim 10^{15} \mathrm{~T}\right)$ can be produced (mostly by energetic spectator protons) $[2,3]$ and will induce an electric current along $\vec{B}$ in chiral domains, $\vec{J}_{e} \propto \mu_{5} \vec{B}$, which is called the chiral magnetic effect (CME) $[1,2]$. On average, $\vec{B}$ is perpendicular to the so-called reaction plane $\left(\Psi_{\mathrm{RP}}\right)$ that contains the impact parameter and the beam momenta, as depicted in Figure 1. Hence the CME will manifest a charge transport across the reaction plane.

In the presence of the CME and other modes of collective motions, we can Fourier decompose the azimuthal distribution of particles of given transverse momentum $\left(p_{T}\right)$ and pseudorapidity $(\eta)$ :

$$
\begin{aligned}
\frac{d N_{\alpha}}{d \varphi} \propto & 1+2 v_{1, \alpha} \cos (\Delta \varphi)+2 v_{2, \alpha} \cos (2 \Delta \varphi)+\cdots \\
+ & 2 a_{1, \alpha} \sin (\Delta \varphi)+\cdots,
\end{aligned}
$$

where $\varphi$ is the azimuthal angle of a particle and $\Delta \varphi=\varphi-$ $\Psi_{\mathrm{RP}}$. Here the subscript $\alpha$ (+ or - ) denotes the charge sign of the particle. Conventionally $v_{1}$ is called "directed flow" and $v_{2}$ "elliptic flow" [7]. The parameter $a_{1}$ (with $a_{1,-}=-a_{1,+}$ ) quantifies the electric charge separation due to the CME.

An anomalous transport effect can also occur when a chiral system undergoes a global rotation. The fluid rotation can be quantified by a vorticity $\vec{\omega}=\vec{\nabla} \times \vec{v}$, where $\vec{v}$ is the flow velocity field. For a given vorticity $\vec{\omega}$, the chiral vortical effect (CVE) induces a vector current $\vec{J}_{v} \propto \mu_{5} \mu_{v} \vec{\omega}$ [8]. Whereas the CME is driven by $\vec{B}$, the CVE is driven by $\mu_{v} \vec{\omega}$ in a chiral medium. Here the subscript " $v$ " means "vector," and can be, for example, " $B$ " (baryon) or " $e$ " (electron). In heavy-ion collisions, $\mu_{B}$ is typically larger than $\mu_{e}$ by an order of magnitude, making it easier to search for the CVE via the baryonic-charge separation than the electric charge separation. Hence the subscript $\alpha$ in (1) represents baryon or antibaryon in the CVE search.

Another complementary transport phenomenon to the CME has been found and named the chiral separation effect (CSE) $[9,10]$, in which chiral charges are separated along the 
magnetic field direction in the presence of a finite vectorcharge density: $\vec{J}_{5} \propto \mu_{v} \vec{B}$. In a chirally symmetric phase, the CME and CSE form a collective excitation, the chiral magnetic wave (CMW), a long wavelength hydrodynamic mode of chiral charge densities [11, 12]. The CMW is a signature of the chiral symmetry restoration and manifests itself in a finite electric quadrupole moment of the collision system, where the "poles" ("equator") of the produced fireball acquire additional positive (negative) charge [11]. This effect, if present, will be reflected in the measurements of chargedependent elliptic flow.

There are other chiral magnetic/vortical effects such as the chiral electric separation effect (CESE) [13, 14] and the chiral vortical wave (CVW) [15]; see [16] for a recent review on these effects. This article reviews the experimental results in the past decade to search for the chiral magnetic/vortical effects in high-energy heavy-ion collisions: evidence for the initial magnetic field and vorticity in Section 2, the observation of the electric (baryonic) charge separation in Section 3 (Section 4), and the manifestation of the electric quadrupole moment in Section 5. An outlook for future development is discussed in Section 6.

\section{Driving Force}

We may intuitively regard the magnetic field (vorticity) as the driving force of the CME (CVE), while the chirality imbalance is the initial condition, and the electric (baryonic) charge separation is the manifestation. A rough estimate of the initial magnetic field gives $e B \sim \gamma \alpha_{\mathrm{EM}} Z / b^{2}$, where $\alpha_{\mathrm{EM}} \approx$ $1 / 137, b$ is the impact parameter, and $\gamma$ is the Lorentz factor. Therefore a typical Au+Au collision at $\sqrt{s_{\mathrm{NN}}}=200 \mathrm{GeV}$ produces $e B \sim 1 /\left(1 \mathrm{fm}^{2}\right) \sim m_{\pi}^{2}$. Many computations have attempted to quantify the electromagnetic field on an event-byevent basis (see, e.g., [17-20]), in terms of the spatial distribution, the orientation fluctuation as well as the dependence on colliding nuclei, centrality, and beam energy.

A major uncertainty in theoretical calculations of the magnetic field $\vec{B}$ is its duration in the QCD fluid created in the heavy-ion collision (see, e.g., [21-24]). The time dependence of $\vec{B}$ after the impact of the two nuclei crucially depends on whether/when/how a conducting medium may form and the lifetime of the magnetic field may be elongated. The electric conductivity [25] and the time evolution of the quark densities [26] can be studied via directed flow of charged hadrons in asymmetric collisions, such as $\mathrm{Cu}+\mathrm{Au}$. Figure 2 illustrates the transverse plane for a $\mathrm{Cu}+\mathrm{Au}$ collision with $b$ $=6 \mathrm{fm}$ [27]. The difference in the number of protons creates a strong electric field in the initial stage of the collision, pointing along the arrow in the figure. The lifetime of the electric field might be very short (e.g., $t \sim 0.25 \mathrm{fm} / c$ from $[25$, 26]), but the electric charges from quarks and antiquarks that are present in the early stage of the collision would experience the Coulomb force and lift the degeneracy in $v_{1}$ between positively and negatively charged particles [18, 19, 25]:

$$
v_{1}^{ \pm}=v_{1} \pm d_{E}\left\langle\cos \left(\Psi_{\mathrm{RP}}-\Psi_{E}\right)\right\rangle,
$$

where $\Psi_{E}$ denotes the azimuthal angle of the electric field and the coefficient $d_{E}$ characterizes the strength of the dipole deformation induced by the electric field and is proportional to the electric conductivity of the medium. Here $v_{1}$ represents the rapidity-even component of directed flow that is dominant in asymmetric collisions, whereas in symmetric collisions $v_{1}$ conventionally denotes the rapidityodd component.

Figure 3 shows recent STAR measurements of chargedependent $v_{1}{ }^{\text {even }}$ and the difference $\Delta v_{1}{ }^{\text {even }}$ as functions of $p_{T}$ in $10-40 \% \mathrm{Cu}+\mathrm{Au}$ and $\mathrm{Au}+\mathrm{Au}$ collisions [27]. For $p_{T}<$ $2 \mathrm{GeV} / c, \Delta v_{1}{ }^{\text {even }}$ seems to increase with $p_{T}$. The $v_{1}{ }^{\text {even }}$ results from $\mathrm{Au}+\mathrm{Au}$ collisions have much smaller values, roughly by a factor of 10 , than those in $\mathrm{Cu}+\mathrm{Au}$. Note that $v_{1}{ }^{\text {odd }}$ in $\mathrm{Au}+\mathrm{Au}$ collisions is similarly small [28]. $\Delta v_{1}{ }^{\text {even }}$ in $\mathrm{Au}+\mathrm{Au}$ is consistent with zero. Calculations for charged pions from the parton-hadron-string-dynamics (PHSD) model [26], which is a dynamical transport approach in the partonic and hadronic phases, are compared with the data. The PHSD model calculates two cases: charge-dependent $v_{1}{ }^{\text {even }}$ with and without the initial electric field (EF). In the case with the EF switched on, the model assumes that all electric charges are affected by the EF and this results in a large separation of $v_{1}{ }^{\text {even }}$ between positive and negative particles as shown in Figure 3(a). In Figure 3(b), the calculations of $v_{1}{ }^{\text {even }}$ with and without the EF are shown together, but note that the EF-on calculation points are scaled by 0.1 . After scaling by 0.1 , the model describes rather well the $p_{T}$ dependence of the measured data for $p_{T}<2 \mathrm{GeV} / c$. This qualitative evidence for the strong initial electric field in asymmetric collisions provides an indirect evidence for the strong initial magnetic field in heavy-ion collisions that could leave an imprint on the finalstage particles.

The vorticity is induced by the global rotation of the QGP in heavy-ion collisions. In a noncentral collision, the majority of the global angular momentum, $\vec{L}$, is carried away by spectator nucleons. However, a considerable fraction (about $10-20 \%$ ) of $\vec{L}$ could remain in the QGP and be approximately conserved in time $[29,30]$. This implies a relatively long duration of the vortical effects. On average, the angular momentum is pointing in the out-of-plane direction, so the CME and CVE are very much alike in terms of their experimental observables. Attempts to compute local vorticity $\vec{\omega}$ and its space-time distribution have also been made [29-34].

Experimentally global polarization of hyperons such as $\Lambda$ provides a measure for both the plasma vorticity and the magnetic field. Whereas the vortical effects will generate a positive polarization for both $\Lambda$ and $\bar{\Lambda}$, the coupling of the hadronic magnetic dipole moment to the magnetic field will generate a positive contribution for $\Lambda$ and a negative one for $\bar{\Lambda}$. Therefore, a splitting between $\Lambda$ and $\bar{\Lambda}$ polarization will be a direct evidence for the strong initial magnetic field. Recently, preliminary STAR measurements [35] have reported the first observation of global $\Lambda$ and $\bar{\Lambda}$ polarization in heavy-ion collisions. At $\sqrt{s_{\mathrm{NN}}}<100 \mathrm{GeV}$, the signal is on the order of a few percent and displays a weak beam energy dependence. The average polarization over $\Lambda$ and $\bar{\Lambda}$ evidences the plasma vorticity, while the splitting observation requires 


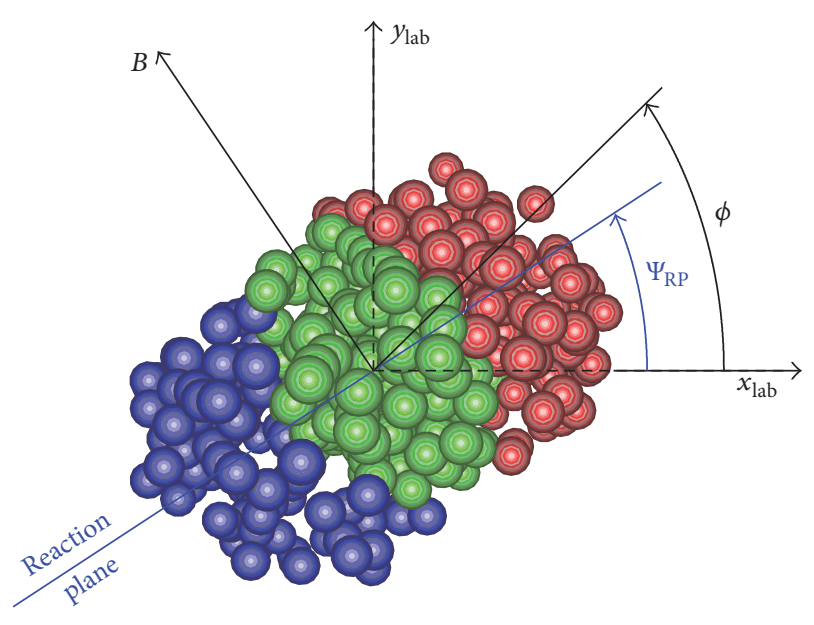

FIGURE 1: Schematic depiction of the transverse plane for a collision of two heavy ions (the left one emerging from and the right one going into the page). Particles are produced in the overlap region (green-colored nucleons). The azimuthal angles of the reaction plane and a produced particle are depicted here.

much higher statistics to be delivered in the second beam energy scan (BES-II) program at RHIC [36] to signify the magnetic field.

The future search for evidence for the initial magnetic field (vorticity) can be conducted via photon (vector meson) polarization measurements [37]. The initial magnetic helicity $(\vec{E} \cdot \vec{B})$ of the collision system can be quite large and bears opposite signs in the upper and lower hemispheres. Owing to the chiral anomaly, the helicity can be transferred back and forth between the magnetic flux and fermions as the collision system evolves, so that the magnetic helicity could last long enough to yield photons with opposite circular polarizations in the hemispheres above and below the reaction plane [38-41]. A similar asymmetry in photon polarization can also result from the initial global quark polarization [42], which could effectively lead to a polarization of photons [39]. This local imbalance of photon circular polarization could be observed in experiments, for example, by studying the polarization preference with respect to the reaction plane for photons that convert into $e^{+} e^{-}$pairs [37]. Similarly, vector mesons that decay into two daughters can also have their polarization preferences measured with the scheme outlined in [37], and the helicity separation in this case originates from vorticity [42-44].

\section{Chiral Magnetic Effect}

In high-energy heavy-ion collisions, the occurrence of the CME requires the simultaneous existence of ultra-strong magnetic fields, chiral symmetry restoration, and topological charge changing transitions. Therefore, the confirmation of the CME will further our understanding of all these fundamental physics mechanisms. The experimental searches for the CME have been carried out extensively in the past decade at RHIC and the LHC. This section will introduce the observables pertinent to the electric charge separation

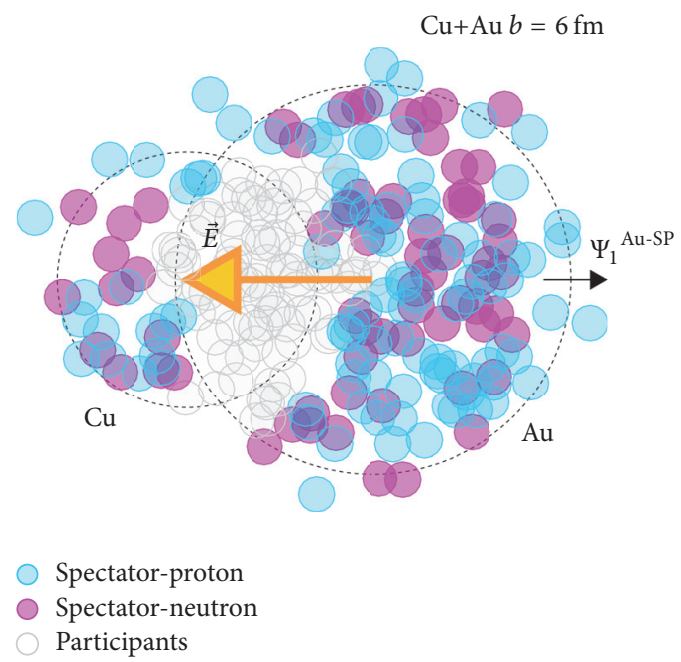

Figure 2: Example of a noncentral $\mathrm{Cu}+\mathrm{Au}$ collision viewed in the transverse plane showing an initial electric field $\vec{E}$ caused by the charge difference between the two nuclei [27]. $\Psi_{1}^{\text {Au-SP }}$ denotes the direction of Au spectators.

induced by the CME, present the experimental results, and discuss the background contributions due to the coupling of elliptic flow and other physics mechanisms.

3.1. Charge Separation Observable. It is tempting to directly measure the event average of $a_{1, \pm}$. However, from event to event, the signs of the $\mu_{5}$ values are equally likely, and the signs of finite $a_{1,+}$ and $a_{1,-}$ will flip accordingly, which will result in $\left\langle a_{1,+}\right\rangle=\left\langle a_{1,-}\right\rangle=0$. Figure 4 presents the STAR measurements of $\left\langle a_{1, \pm}\right\rangle$ with the 1st harmonic event plane reconstructed from spectator neutrons [45]. These results indicate nonsignificant charge dependence in all centrality intervals, and the difference between positive and negative charges is typically less than $10^{-4}$.

One solution to search for the CME is via charge separation fluctuations perpendicular to the reaction plane, for example, with a three-point correlator [55], $\gamma \equiv\left\langle\left\langle\cos \left(\varphi_{\alpha}+\right.\right.\right.$ $\left.\left.\left.\varphi_{\beta}-2 \Psi_{\mathrm{RP}}\right)\right\rangle\right\rangle$, where the averaging is done over all particles in an event and over all events. In practice, the reaction plane is approximated with the "event plane" $\left(\Psi_{\mathrm{EP}}\right)$ reconstructed with measured particles, and then the measurement is corrected for the finite event plane resolution. The expansion of the $\gamma$ correlator,

$$
\begin{aligned}
\gamma & \equiv\left\langle\left\langle\cos \left(\varphi_{\alpha}+\varphi_{\beta}-2 \Psi_{\mathrm{RP}}\right)\right\rangle\right\rangle \\
& =\left\langle\left\langle\cos \left(\Delta \varphi_{\alpha}\right) \cos \left(\Delta \varphi_{\beta}\right)-\sin \left(\Delta \varphi_{\alpha}\right) \sin \left(\Delta \varphi_{\beta}\right)\right\rangle\right\rangle \\
& =\left(\left\langle v_{1, \alpha} v_{1, \beta}\right\rangle+B_{\mathrm{IN}}\right)-\left(\left\langle a_{1, \alpha} a_{1, \beta}\right\rangle+B_{\mathrm{OUT}}\right),
\end{aligned}
$$

reveals the difference between the in-plane and out-of-plane projections of the correlations. The first term $\left(\left\langle v_{1, \alpha} v_{1, \beta}\right\rangle\right)$ in the expansion provides a baseline unrelated to the magnetic field. The background contribution $\left(B_{\mathrm{IN}}-B_{\mathrm{OUT}}\right)$ is suppressed to a level close to the magnitude of $v_{2}$ [55]. 

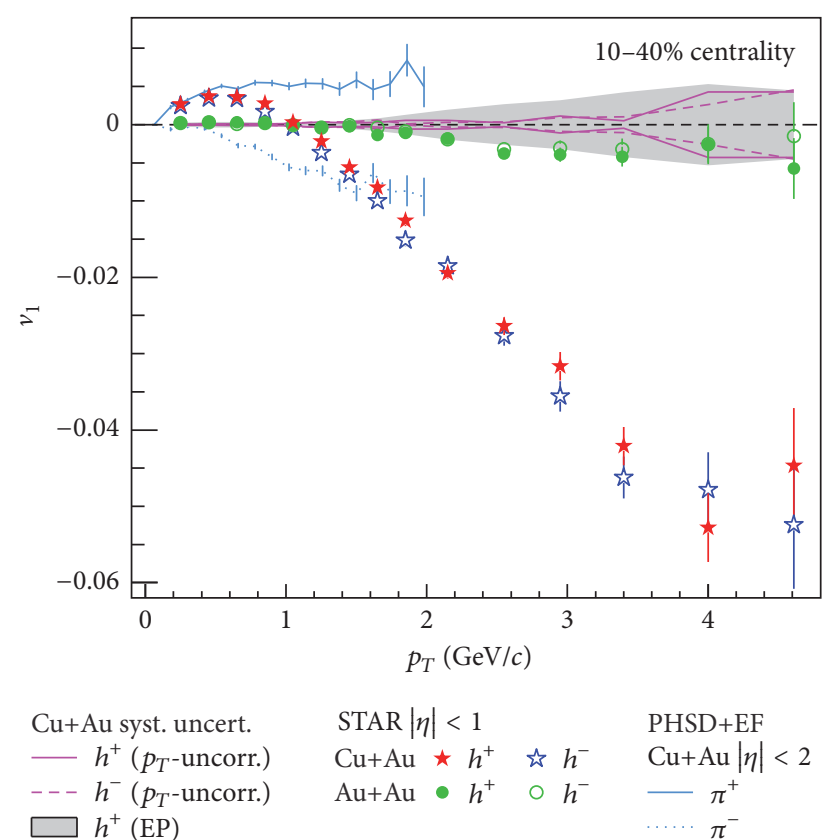

(a)

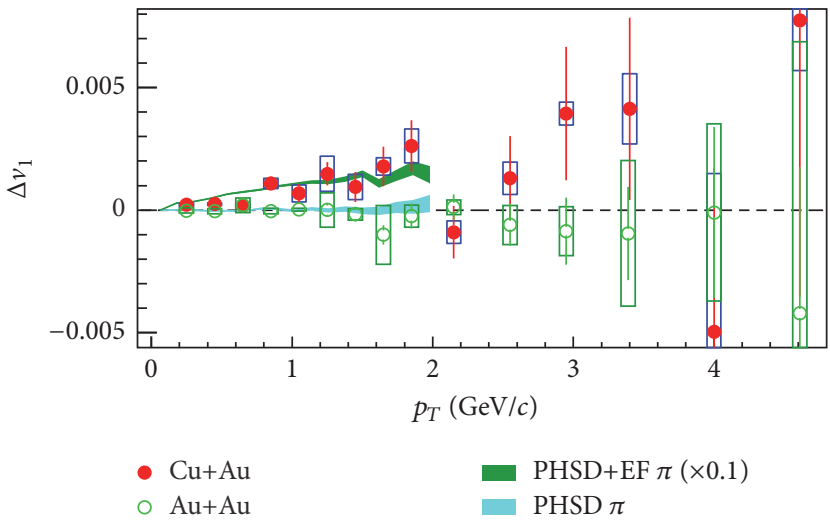

(b)

FIGURE 3: $v_{1}$ even of positive and negative particles and the difference between the two as functions of $p_{T}$ in $10-40 \% \mathrm{Cu}+\mathrm{Au}$ and $\mathrm{Au}+\mathrm{Au}$ collisions [27]. The PHSD model calculations [26] for charged pions with and without the initial electric field (EF) are presented for comparison.

The $\gamma$ correlator was first measured by the STAR Collaboration with the 2 nd harmonic event plane for $\mathrm{Au}+\mathrm{Au}$ (shown with crosses in Figure 5) and $\mathrm{Cu}+\mathrm{Cu}$ (not shown here) collisions at 62.4 and $200 \mathrm{GeV}$ with data from the 2004/2005 RHIC runs [46, 47]. All the results have been found to be in qualitative expectation with the CME: the opposite-charge $\left(\gamma_{\mathrm{OS}}\right)$ is above the same-charge $\left(\gamma_{\mathrm{SS}}\right)$ correlations, displaying a "right" ordering. The opposite-charge correlations in $\mathrm{Cu}+\mathrm{Cu}$ collisions are stronger than those in $\mathrm{Au}+\mathrm{Au}$, possibly because of the suppression of the correlations among oppositely moving particles in a larger system. STAR also presented $p_{T}$ and $\Delta \eta$ dependences of the signal. The signal has a $\Delta \eta$ width of about one unit of rapidity, suggesting that shortrange correlations are not the dominant contributions. The

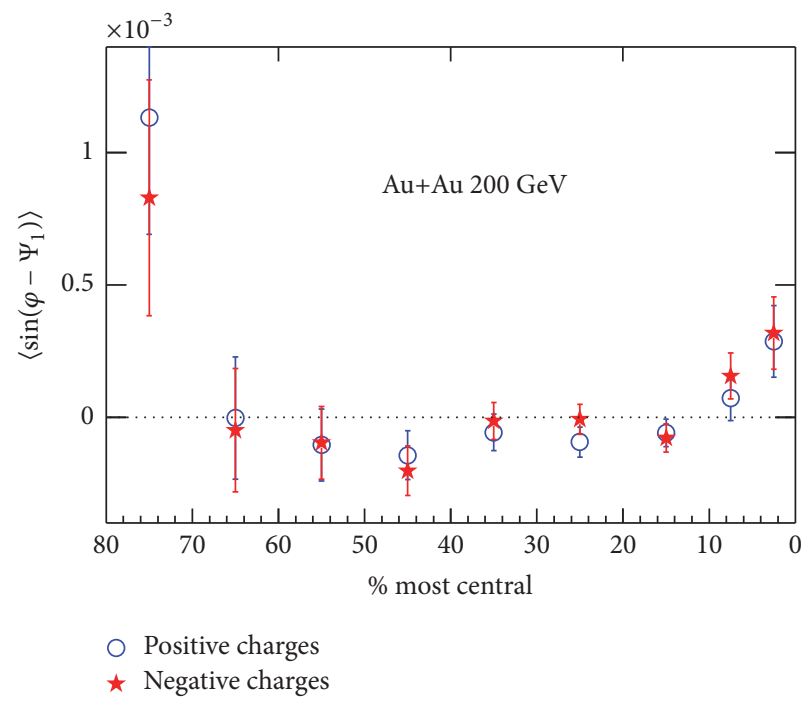

Figure 4: $\left\langle\sin \left(\varphi-\Psi_{1}\right)\right\rangle$ for positive and negative charges versus centrality for $\mathrm{Au}+\mathrm{Au}$ collisions at $\sqrt{s_{\mathrm{NN}}}=200 \mathrm{GeV}$ [45].

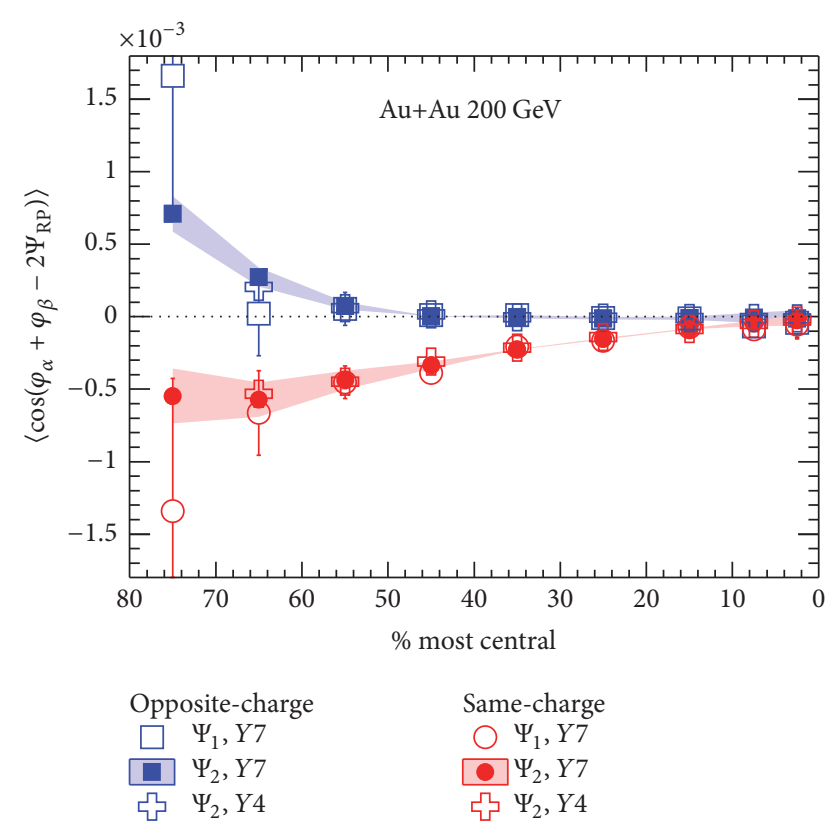

FIGURE 5: Three-point correlator, $\gamma$, measured with 1st and 2nd harmonic event planes versus centrality for $\mathrm{Au}+\mathrm{Au}$ collisions at $\sqrt{s_{\mathrm{NN}}}=200 \mathrm{GeV}$ [45]. Shown with crosses are STAR previous results from the 2004 RHIC run $[46,47]$.

signal is found to increase with the pair average $p_{T}$, and it was later shown [56] that the radial flow expansion can explain this feature.

Similar $\gamma$ results for $200 \mathrm{GeV} \mathrm{Au}+\mathrm{Au}$ and $2.76 \mathrm{TeV} \mathrm{Pb}+\mathrm{Pb}$ were observed by the PHENIX Collaboration [57] and the ALICE Collaboration [49], respectively. PHENIX also employed a multiparticle charge-sensitive correlator, $\operatorname{Cc}(\Delta S)$ [58], and their preliminary results showed a concave $\operatorname{Cc}(\Delta S)$ distribution, also evidencing the charge separation effect. Recently this method was also applied to the STAR data, 
which yielded qualitatively similar results [59]. However, there are a few issues with this method that keep us from further discussing its results. First, this correlator employs charge-sign-randomized particles to provide a baseline for the charge separation, but the randomization will introduce a bias by destroying local charge conservation. Second, there is no correction for the event plane resolution in the measurement, making it impossible to directly compare the measurements between different experiments. Third, the extraction of the charge separation signal relies on the comparison with a simplified Monte Carlo simulation, which has to well describe many experimental phenomena, including $v_{2}$, spectra, event plane resolution, transverse momentum conservation, and local charge conservation. Among other things, we are not aware of any simple Monte Carlo simulation that properly handles momentum conservation. In short, the reliability of this method depends on how realistic the simple simulation can be.

The conventional-physics backgrounds were investigated by employing heavy-ion event generators MEVSIM [50], UrQMD [60], and HIJING [61] (with and without an elliptic flow afterburner implemented). None of these generators could achieve reasonable agreement with the data. However, these generators do not well describe main features of heavyion collisions, so their failure to describe effects as subtle as charge separation provides only limited support to the CME picture.

The charge separation signal was cross-checked with data from the 2007 RHIC run (shown in Figure 5) [45]. The $\gamma$ correlations from these data were measured with respect to both the 1st harmonic event plane (of spectators at large rapidity) and the 2 nd harmonic event planes at midrapidity. The agreement of results using different types of event planes ensures that the signal is not caused by threeparticle background correlations and is truly due to reactionplane-related correlations.

Another test was carried out by replacing one of the two charged particles in $\gamma$ with a neutral particle, for example, $K_{S}{ }^{0}$, and the results show no separation between $K_{S}{ }^{0}-h^{+}$and $K_{S}^{0}-h^{-}$[62]. Thus the charge separation observed in the $\gamma$ correlation between two charged particles is indeed due to the electric charge. Short-range correlations are visible in the differential measurements of $\gamma$ at low relative momenta, which are related to quantum interference ("HBT") and final-stateinteractions (Coulomb dominated) [45], and they belong to late-stage effects. To suppress short-range correlations, the conditions of $\Delta p_{T}>0.15 \mathrm{GeV} / c$ and $\Delta \eta>0.15$ were applied to the three-point correlator, shown with the gray bars in Figure 6. When pairs with low relative momenta are excluded, the positive opposite-charge correlations in peripheral collisions are significantly reduced, but the difference between same- and opposite-charge correlations remains largely unchanged.

Different azimuthal regions of charge separation are weighted differently in the $\gamma$ correlator; that is, oppositely charged pairs emitted azimuthally at $90^{\circ}$ from the event plane (maximally out-of-plane) are weighted more heavily than those emitted only a few degrees from the event plane

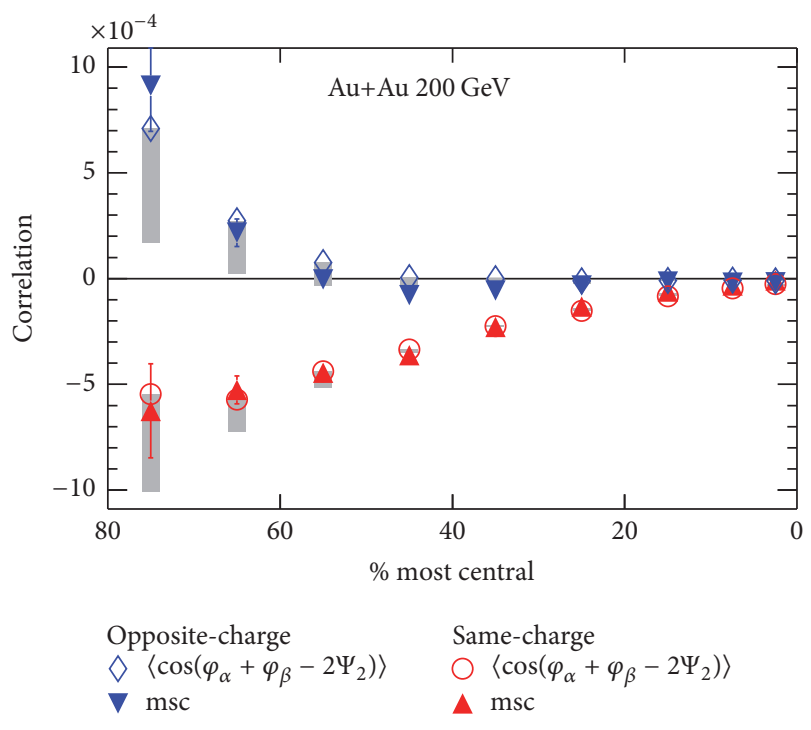

FIGURE 6: Modulated sign correlations (msc) compared to the threepoint correlator versus centrality for $\mathrm{Au}+\mathrm{Au}$ collisions at $\sqrt{s_{\mathrm{NN}}}$ $=200 \mathrm{GeV}$ [45]. The gray bars reflect the conditions of $\Delta p_{T}>$ $0.15 \mathrm{GeV} / c$ and $\Delta \eta>0.15$ applied to $\gamma$.

(minimally out-of-plane). A test was carried out to modify the $\gamma$ correlator such that all azimuthal regions of charge separation receive an identical weight. This may be done by first rewriting (3) as

$$
\begin{aligned}
& \left\langle\cos \left(\varphi_{\alpha}+\varphi_{\beta}-2 \Psi_{\mathrm{RP}}\right)\right\rangle \\
& =\left\langle\left(M_{\alpha} M_{\beta} S_{\alpha} S_{\beta}\right)_{\mathrm{IN}}-\left(M_{\alpha} M_{\beta} S_{\alpha} S_{\beta}\right)_{\mathrm{OUT}}\right\rangle,
\end{aligned}
$$

where $M$ and $S$ stand for the absolute magnitude $(0 \leq M \leq 1)$ and the sign $( \pm 1)$ of the sine or cosine function, respectively. IN represents the cosine part of (3) (in-plane), and OUT represents the sine part (out-of-plane). A modulated sign correlation (msc) is obtained by reducing the $\gamma$ correlator [45]:

$$
\mathrm{msc} \equiv\left(\frac{\pi}{4}\right)^{2}\left(\left\langle S_{\alpha} S_{\beta}\right\rangle_{\mathrm{IN}}-\left\langle S_{\alpha} S_{\beta}\right\rangle_{\mathrm{OUT}}\right) .
$$

The modulated sign correlations are compared with the threepoint correlator for $\mathrm{Au}+\mathrm{Au}$ collisions at $200 \mathrm{GeV}$ in Figure 6 . The msc turns out to well reproduce the same trend as the three-point correlator although their magnitudes differ slightly. STAR also invented another approach called the charge multiplicity asymmetry correlation (CMAC), whose methodology is similar to the $\mathrm{msc}$, and yielded very similar results [63].

The beam energy dependence of the correlation could further our understanding of the origin of the observed charge separation. The charge separation effect depends strongly on the formation of the QGP and chiral symmetry restoration [1], so the signal will diminish if a QGP is short-lived or even absent at low collision energies. The fact that the lifetime of the magnetic field is longer at smaller collision energies could lead to an almost threshold effect: 


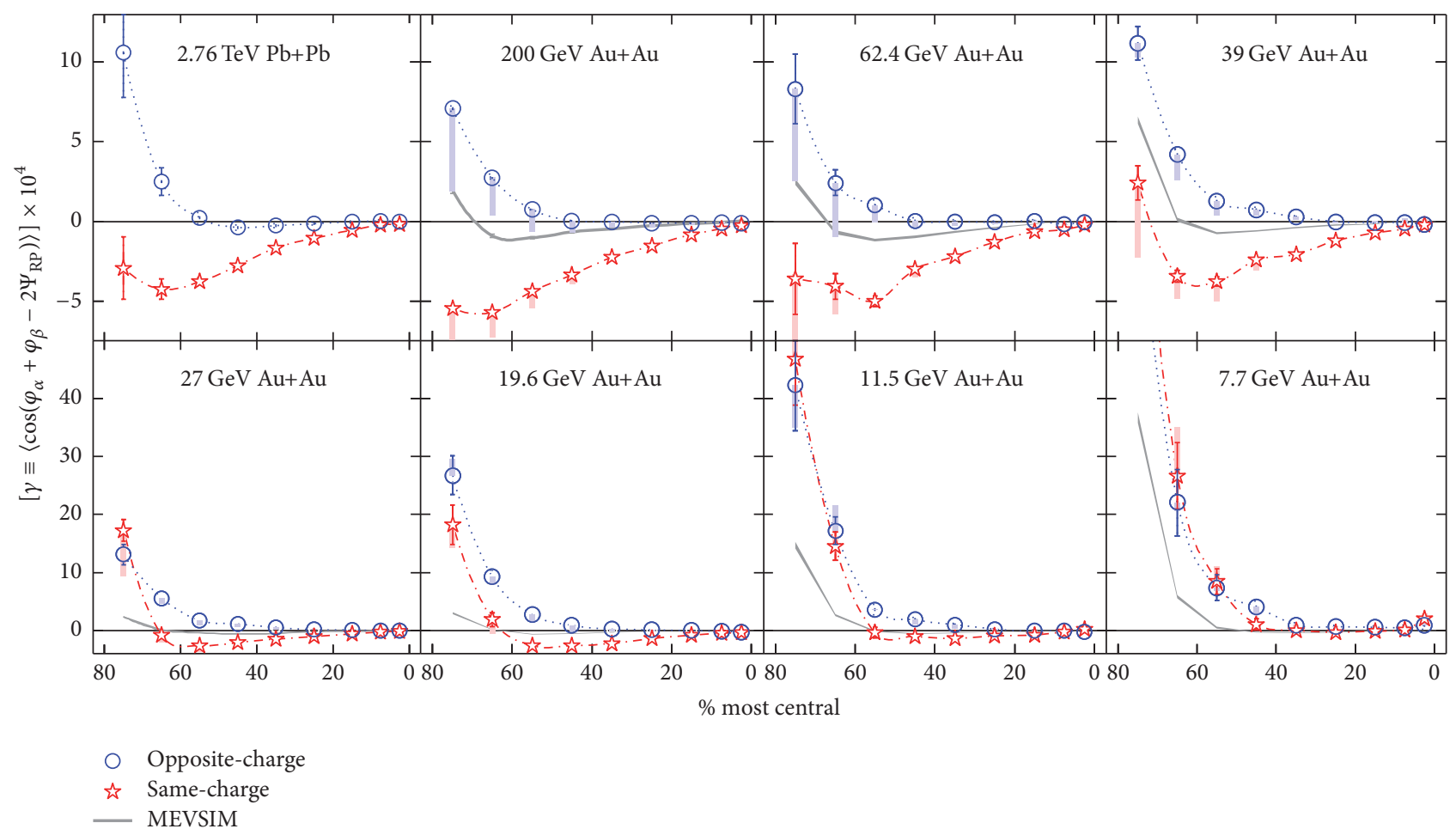

Figure 7: Three-point correlator as a function of centrality for $\mathrm{Au}+\mathrm{Au}$ collisions at $7.7-200 \mathrm{GeV}$ [48] and for $\mathrm{Pb}+\mathrm{Pb}$ collisions at $2.76 \mathrm{TeV}$ [49]. Note that the vertical scales are different for different rows. The systematic errors (gray bars) bear the same meaning as in Figure 6. Charge-independent results from the model calculations of MEVSIM [50] are shown as gray curves.

with decreasing collision energy, the signal might slowly increase with an abrupt drop thereafter. Unfortunately, the exact energy dependence of the CME is not calculated yet.

Figure 7 presents $\gamma_{\mathrm{OS}}$ and $\gamma_{\mathrm{SS}}$ correlators as functions of centrality for $\mathrm{Au}+\mathrm{Au}$ collisions at $\sqrt{s_{\mathrm{NN}}}=7.7-200 \mathrm{GeV}$ measured by STAR [48] and for $\mathrm{Pb}+\mathrm{Pb}$ collisions at $2.76 \mathrm{TeV}$ by ALICE [49]. In most cases, $\gamma_{\mathrm{OS}}$ is still above $\gamma_{\mathrm{SS}}$, manifesting extra charge separation fluctuations perpendicular to the reaction plane. With decreased beam energy, both $\gamma_{\mathrm{OS}}$ and $\gamma_{\mathrm{SS}}$ tend to rise up starting from peripheral collisions. This feature seems to be charge-independent and can be explained by momentum conservation and elliptic flow [45]. Momentum conservation forces all produced particles, regardless of charge, to separate from each other, whereas elliptic flow works in the opposite sense. In peripheral collisions, momentum conservation dominates because the multiplicity $(N)$ is small. The lower beam energy, the smaller $N$, and the higher $\gamma_{\mathrm{OS}}$ and $\gamma_{\mathrm{SS}}$. In more central collisions where the multiplicity is large enough, this type of charge-independent background can be described by $-v_{2} / N[45,64]$. MEVSIM is a Monte Carlo event generator developed for STAR simulations [50]. In Figure 7, we also show the model calculations of MEVSIM with the implementation of $v_{2}$ and momentum conservation, which qualitatively describe the beam energy dependence of the charge-independent background. The difference between $\gamma_{\mathrm{OS}}$ and $\gamma_{\mathrm{SS}}$ seems to vanish at low collision energies, but the interpretation involves an ambiguity to be discussed in Section 3.2.
3.2. Flow Backgrounds. By construction, the $\gamma$ correlator contains the background terms $B_{\mathrm{IN}}$ and $B_{\mathrm{OUT}}$, and their difference was originally studied for the "flowing cluster" case [55]:

$$
\frac{B_{\mathrm{IN}}-B_{\mathrm{OUT}}}{B_{\mathrm{IN}}+B_{\mathrm{OUT}}} \approx v_{2, \mathrm{cl}} \frac{\left\langle\cos \left(\varphi_{\alpha}+\varphi_{\beta}-2 \varphi_{\mathrm{cl}}\right)\right\rangle}{\left\langle\cos \left(\varphi_{\alpha}-\varphi_{\beta}\right)\right\rangle},
$$

where $\varphi_{\mathrm{cl}}$ is the cluster emission azimuthal angle and $\varphi_{\alpha}$ and $\varphi_{\beta}$ are the azimuthal angles of two decay products. The flowing cluster can be generalized to a larger portion of or even the whole event, through the mechanisms of transverse momentum conservation (TMC) $[65,66]$ and/or local charge conservation (LCC) [67]. We introduce a useful tool to study the background, the two-particle correlator, $\delta \equiv\left\langle\cos \left(\varphi_{\alpha}-\right.\right.$ $\left.\left.\varphi_{\beta}\right)\right\rangle$. Ideally $\delta$ should be proportional to $\left\langle a_{1, \alpha} a_{1, \beta}\right\rangle$, but in reality it is dominated by backgrounds. For example, the TMC effect leads to the following pertinent correlation terms in $\delta$ and $\gamma[66]$ :

$$
\begin{aligned}
& \delta \longrightarrow-\frac{1}{N} \frac{\left\langle p_{T}\right\rangle_{\Omega}^{2}}{\left\langle p_{T}^{2}\right\rangle_{F}} \frac{1+\bar{v}_{2, \Omega}^{2}-2 \overline{\bar{v}}_{2, F} \bar{v}_{2, \Omega}}{1-\overline{\bar{v}}_{2, F}^{2}}, \\
& \gamma \longrightarrow-\frac{1}{N} \frac{\left\langle p_{T}\right\rangle_{\Omega}^{2}}{\left\langle p_{T}^{2}\right\rangle_{F}} \frac{2 \bar{v}_{2, \Omega}-\overline{\bar{v}}_{2, F}-\overline{\bar{v}}_{2, F} \bar{v}_{2, \Omega}^{2}}{1-\overline{\bar{v}}_{2, F}^{2}} \approx \kappa \cdot v_{2, \Omega} \cdot \delta,
\end{aligned}
$$

where $\kappa=\left(2 \bar{v}_{2, \Omega}-\overline{\bar{v}}_{2, F}\right) / v_{2, \Omega}$ and $\bar{v}_{2}$ and $\overline{\bar{v}}_{2}$ represent the $p_{T^{-}}$and $p_{T^{-}}^{2}$-weighted moments of $v_{2}$, respectively. The 


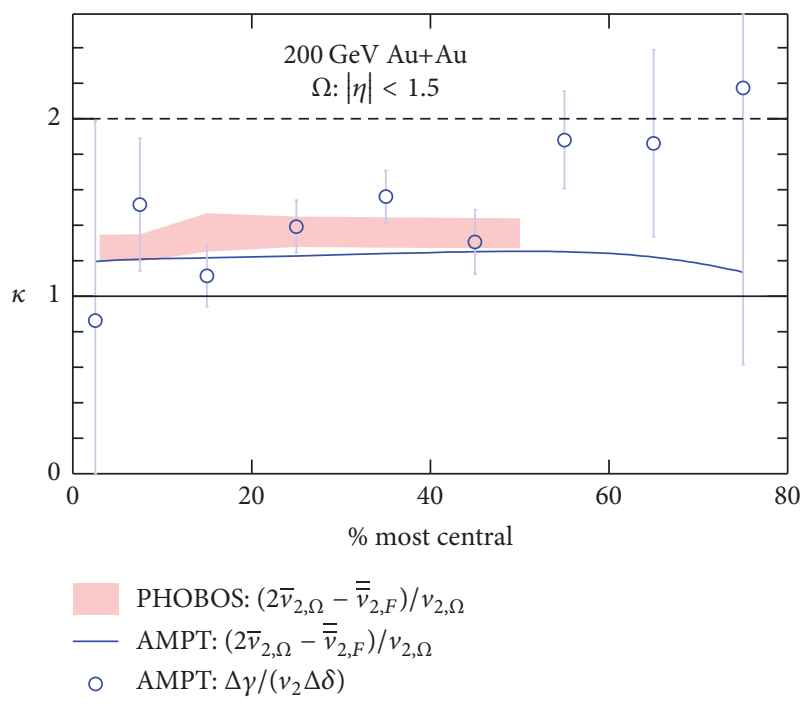

FIGURE 8: Estimation of $\kappa$ with three approaches for $200 \mathrm{GeV} \mathrm{Au}+\mathrm{Au}$ [51].

subscript " $F$ " denotes an average of all produced particles in the full phase space; the actual measurements will be only in a fraction of the full space, denoted by " $\Omega$." The background contribution due to the LCC effect has a similar characteristic structure as the above $[65,67]$.

It is convenient to express $\gamma$ and $\delta$ with a two-component framework $[48,66]$ :

$$
\begin{aligned}
& \gamma \equiv\left\langle\left\langle\cos \left(\varphi_{\alpha}+\varphi_{\beta}-2 \Psi_{\mathrm{RP}}\right)\right\rangle\right\rangle=\kappa v_{2} B-H, \\
& \delta \equiv\left\langle\left\langle\cos \left(\varphi_{\alpha}-\varphi_{\beta}\right)\right\rangle\right\rangle=B+H,
\end{aligned}
$$

where $H$ and $B$ are the CME and background contributions, respectively. The background-subtracted correlator, $H$, can be obtained from the ensemble averages of several observables:

$$
H^{\kappa}=\frac{\left(\kappa v_{2} \delta-\gamma\right)}{\left(1+\kappa v_{2}\right)} \text {. }
$$

The major uncertainty in the above expression, the coefficient $\kappa$, depends on particle charge combination, particle transverse momentum, centrality, and collision energy.

Figure 8 shows the $\kappa$ values estimated for $\mathrm{Au}+\mathrm{Au}$ collisions at $200 \mathrm{GeV}$ [51], with the $v_{2}$ measurements by the PHOBOS collaboration $[68,69]$, and with the $v_{2}$ calculations from the AMPT model [70-72]. Here only the TMC effect has been taken in account, and $\kappa$ is typically within $[1.2,1.4]$ for $|\eta|<1.5$. The $\kappa$ values attained this way will vary slightly if a smaller $|\eta|$ acceptance is involved. In reality, $\kappa$ should be averaged over various mechanisms such as TMC, LCC, and resonance decays. The AMPT model gives a more comprehensive estimate in Figure 8 via $\Delta \gamma /\left(v_{2} \Delta \delta\right)$, where the numerator is solely due to flow backgrounds. For the centrality range of $10-50 \%$, where the statistical uncertainties are small, the $\kappa$ values thus obtained are close to those estimated with the $v_{2}$ information.

Figure 9 shows $\left(H_{\mathrm{SS}}{ }^{\kappa=1}-H_{\mathrm{OS}}{ }^{\kappa=1}\right)$ as a function of beam energy for $30-60 \% \mathrm{Au}+\mathrm{Au}(\mathrm{Pb}+\mathrm{Pb})$ collisions $[48,49]$.

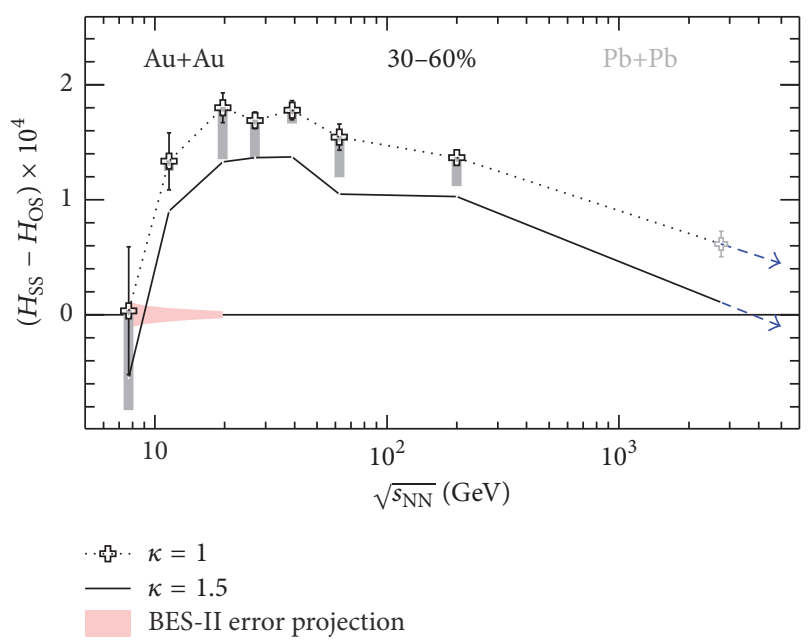

Figure 9: $\Delta H$ as a function of beam energy for 30-60\% $\mathrm{Au}+\mathrm{Au}$ $(\mathrm{Pb}+\mathrm{Pb})$ collisions $[48,49]$. The systematic errors (gray bars) bear the same meaning as in Figure 6.

$\Delta H^{\kappa=1.5}$ is depicted with the solid line. In both cases of $\kappa, \Delta H$ demonstrates a weak energy dependence above $19.6 \mathrm{GeV}$ and tends to diminish from 19.6 to $7.7 \mathrm{GeV}$, though the statistical errors are large for $7.7 \mathrm{GeV}$. This may be explained by the probable domination of hadronic interactions over partonic ones at low energies. A more definitive conclusion may be reached with a more accurate estimation of $\kappa$ and with higher statistics at lower energies in the proposed phase II of the RHIC Beam Energy Scan program, as illustrated by the shaded band in Figure 9. Recently the CMS collaboration carried out the $\gamma$ correlations in $\mathrm{p}+\mathrm{Pb}$ and $\mathrm{Pb}+\mathrm{Pb}$ collisions at $5.02 \mathrm{TeV}$ and observed similar magnitudes of $\Delta \gamma$ versus multiplicity between $\mathrm{p}+\mathrm{Pb}$ and $\mathrm{Pb}+\mathrm{Pb}$ collisions [73]. This seems to indicate no $\mathrm{CME}$ signals in $\mathrm{Pb}+\mathrm{Pb}$ collisions at this collision energy, which could be along the trend shown in Figure 9: a simplified extrapolation to $5.02 \mathrm{TeV}$ points to zero $\Delta H$ if $\kappa$ is around 1.5. A further measurement of the $H$ correlator at $5.02 \mathrm{TeV}$ would help verify/falsify this speculation.

Collisions of Uranium nuclei have been utilized at RHIC to study the dependence of multiplicity production, flow, and the CME on the initial overlap geometry [74, 75]. It was initially thought that the prolate shape of Uranium nuclei would facilitate the selection of nearly fully overlapping events with large elliptic flow values, but with small magnetic fields. However, the square of the magnetic field may be not particularly small, owing to fluctuations. Measurements of very central collisions also demonstrated that the multiplicity does not depend on the configuration of the collisions as strongly as anticipated in the two-component multiplicity model, which significantly reduces the feasibility to independently manipulate the flow and the magnetic field [76].

Figure 10 shows measurements of $\Delta \gamma \equiv \gamma_{\mathrm{OS}}-\gamma_{\mathrm{SS}}$ for the 0 $10 \%$ centrality range in $193 \mathrm{GeV} \mathrm{U}+\mathrm{U}$ (a) and $200 \mathrm{GeV} \mathrm{Au}+\mathrm{Au}$ collisions (b) [52-54]. In both $\mathrm{U}+\mathrm{U}$ and $\mathrm{Au}+\mathrm{Au}$ collisions, the signal increases almost linearly with $v_{2}$, suggesting that 


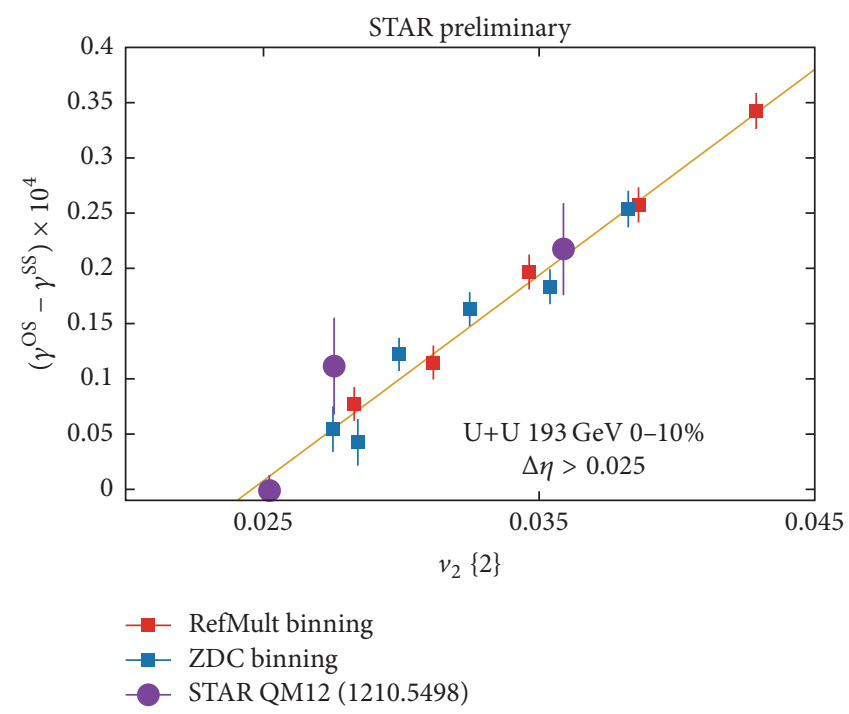

(a)

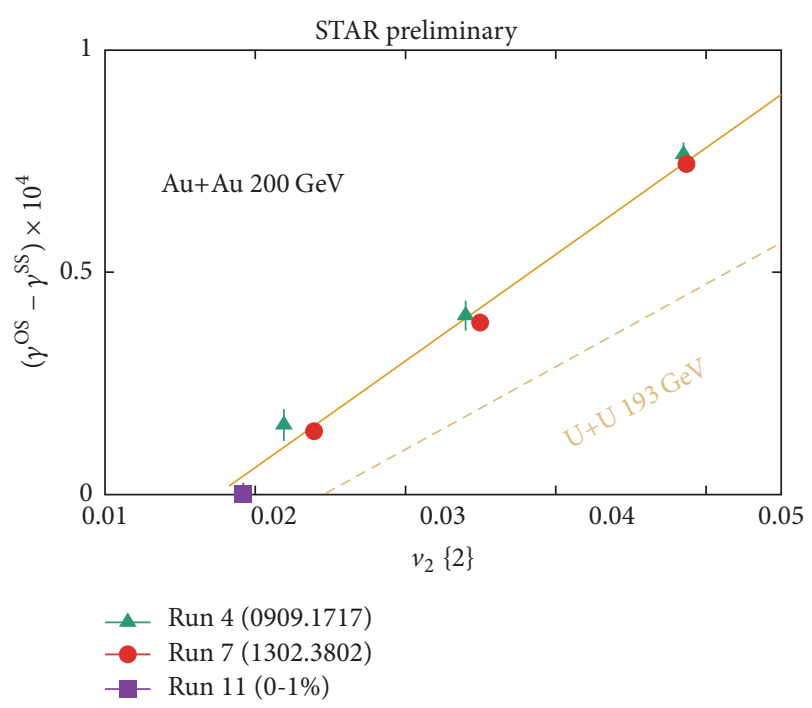

(b)

FIGURE 10: $\Delta \gamma$ as a function of $v_{2}$ for various centrality selections within the $0-10 \%$ centrality range in $\mathrm{U}+\mathrm{U}$ collisions (a) and Au+Au collisions (b) $[52-54]$.

the charge separation observable is dominated by a $v_{2}$ related background. However, the charge separation goes to zero while $v_{2}$ is still sizable in central $\mathrm{Au}+\mathrm{Au}$ and central $\mathrm{U}+\mathrm{U}$ collisions. Model calculations show that the quantity $\left\langle\left(e B / m_{\pi}^{2}\right)^{2} \cos \left[2\left(\Psi_{B}-\Psi_{\mathrm{RP}}\right)\right]\right\rangle$ as a function of eccentricity exhibits the same trend [53]: although $B^{2}$ remains large owing to fluctuations, $\left\langle\cos \left[2\left(\Psi_{B}-\Psi_{\mathrm{RP}}\right)\right]\right\rangle$ goes to zero as $\Psi_{B}$ and $\Psi_{\mathrm{RP}}$ become decorrelated in very central collisions. In other words, while fluctuations in central collisions keep the participant eccentricity positive-definite, the decorrelation of $\Psi_{B}$ and $\Psi_{\mathrm{RP}}$ drives $\left\langle\left(e B / m_{\pi}^{2}\right)^{2} \cos \left[2\left(\Psi_{B}-\Psi_{\mathrm{RP}}\right)\right]\right\rangle$ to zero. Therefore, the data are in better agreement with a CME picture than a flow background interpretation. A phenomenological study for extrapolating both signal and background from $\mathrm{Au}+\mathrm{Au}$ to $\mathrm{U}+\mathrm{U}$ collisions was done in [77].

Another measurement that exploits the 4th harmonic event plane could clarify the origin of the charge-dependent correlations and the role of the background [78]. The correlations, $\left\langle\cos \left(2 \varphi_{\alpha}+2 \varphi_{\beta}-4 \Psi_{\mathrm{RP}}\right)\right\rangle$, should include the effect of the flow-related background, but not any contribution from the CME. The correlations due to the background in this case are expected to be smaller in magnitude since the 4th harmonic flow is usually weaker than the elliptic flow. The preliminary results of such measurements by ALICE are presented in Figure 11 [79] with separate same-charge and opposite-charge correlations relative to the 4 th harmonic event plane as functions of centrality (a) and the difference between same-charge and opposite-charge correlations with respect to the 2 nd and 4 th harmonic event planes (b). The correlation difference relative to the 4 th harmonic event plane is very small and suggestive of small background contributions. Detailed simulations have to be performed to draw more definite conclusion from this measurement.

\section{Chiral Vortical Effect}

The chiral vortical effect (CVE) is in analog to the CME, and its experimental manifestation is the baryonic-charge separation, instead of the electric charge separation, perpendicular to the reaction plane. Therefore, the three-point $\gamma$ correlator is still applicable, only now between two (anti)baryons. However, if both particles are (anti)protons that carry also electric charges, there will be an ambiguity due to the possible CME signal. The study of the $\gamma$ correlator with an electrically neutral baryon, such as $\Lambda$, will provide more conclusive evidence for the baryonic-charge separation effect.

Although (anti) $\Lambda$ 's are electrically neutral, we still need to answer whether strange quarks behave the same way as up/down quarks in the chiral dynamics during the collision. If the answer is no, then (anti) $\Lambda$ 's may still act like electrically charged particles in the $\gamma$ correlation. Figure 12 shows the $\gamma$ correlation of $\Lambda-h^{+}\left(\bar{\Lambda}-h^{-}\right)$and $\Lambda-h^{-}\left(\bar{\Lambda}-h^{+}\right)$as functions of centrality in $\mathrm{Au}+\mathrm{Au}$ collisions at $200 \mathrm{GeV}$ [62]. In this analysis, (anti)protons are excluded from the charged hadrons in the correlator to avoid any possible CVE contribution. Tentatively assigning a positive (negative) charge to $\Lambda(\bar{\Lambda})$, we observe that the "same-charge" and "opposite-charge" correlations are consistent with each other; hence there is no charge-dependent effect. As mentioned in Section 3.1, the null $K_{S}{ }^{0}-h$ correlations [62] tell that the difference between samecharge and opposite-charge correlations as shown in Figure 5 is truly due to the electric charge, so the null charge separation effect in $\Lambda$ - $h$ manifests no electric charges of (anti) $\Lambda$ 's in the $\gamma$ correlation. Thus strange quarks seem to behave the same way as up/down quarks in the chiral dynamics. Furthermore, the $\Lambda-h$ correlation provides a baseline for the $\Lambda$ - $p$ correlation, and any possible signal in the latter should not come from the CME contribution. 


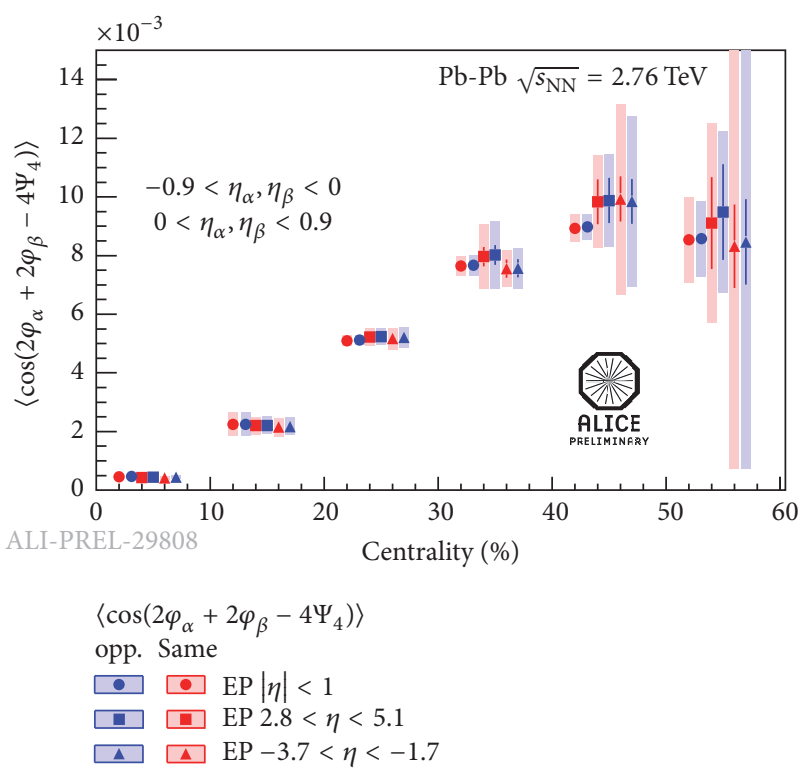

(a)

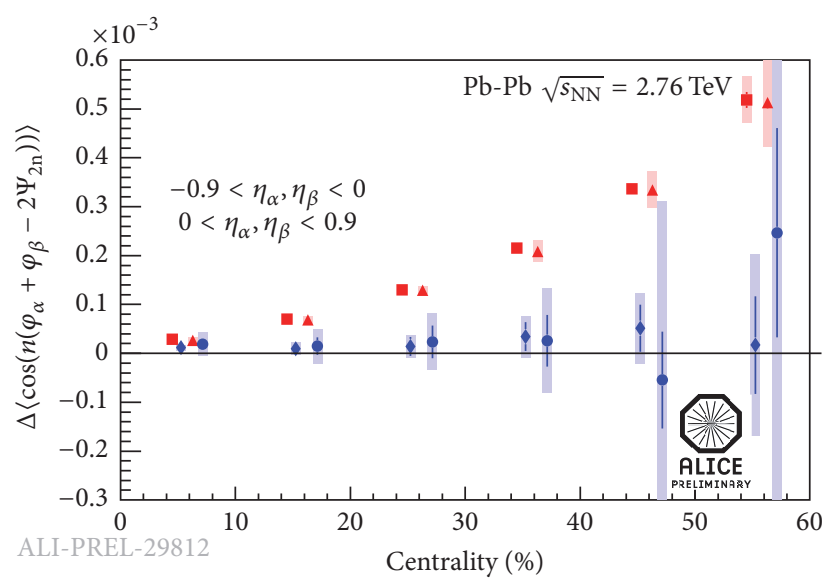

$\mathrm{EP}|\eta|>2 \quad \mathrm{EP}|\eta|<1$

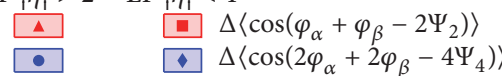

(b)

FIGURE 11: (a) Same-charge and opposite-charge pair correlations relative to the 4th harmonic event plane as functions of centrality. (b) Comparison of the charge-dependent parts in correlations with respect to the 2nd and 4th harmonic event planes [79].

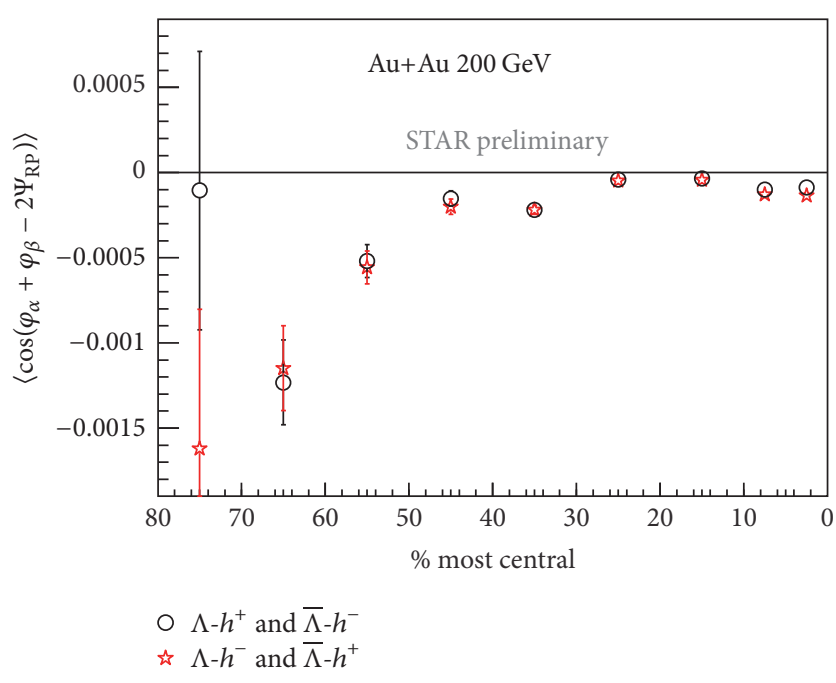

Figure 12: $\gamma$ correlations of $\Lambda-h^{+}\left(\bar{\Lambda}-h^{-}\right)$and $\Lambda-h^{-}\left(\bar{\Lambda}-h^{+}\right)$as functions of centrality in $\mathrm{Au}+\mathrm{Au}$ collisions at $200 \mathrm{GeV}$ [62].

Figure 13 shows $\gamma$ correlations of $\Lambda-p(\bar{\Lambda}-\bar{p})$ and $\Lambda-\bar{p}$ $(\bar{\Lambda}-p)$ as functions of centrality in $\mathrm{Au}+\mathrm{Au}$ collisions at $200 \mathrm{GeV}$ [62]. The same-baryonic-charge correlation is above the opposite-baryonic-charge correlation from mid-central to peripheral collisions. This baryonic-charge separation with respect to the event plane is consistent with the CVE picture. More investigations into the background contribution are needed. For example, in analog to the local charge conservation, the local baryonic-charge conservation could play a similar role as LCC when coupled to $v_{2}$. The magnitudes of the $\Lambda$ - $p$ correlations are much larger than those of the $h-h$ correlations. This is partially because $\left\langle p_{T}\right\rangle$ of baryons

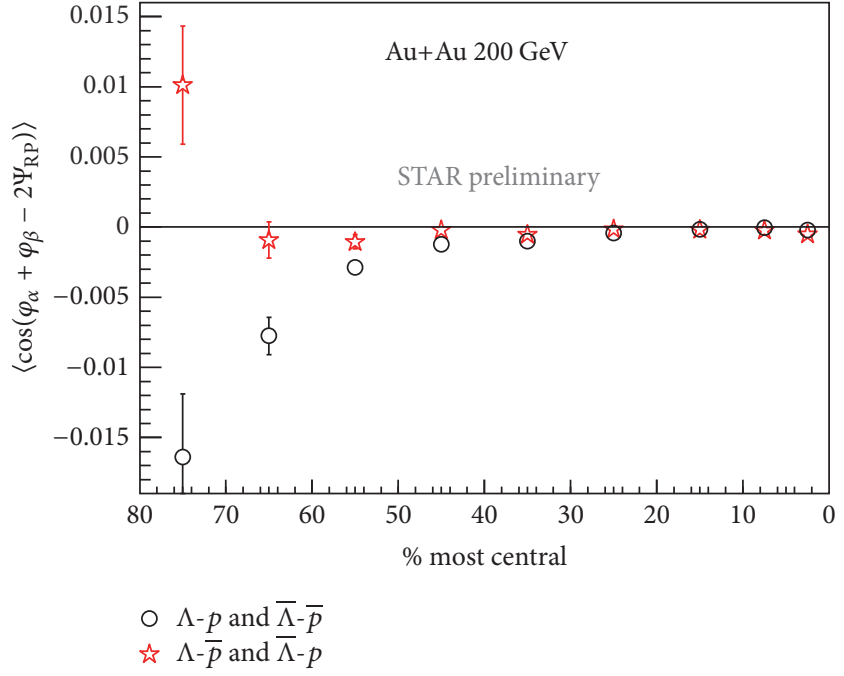

Figure 13: $\gamma$ correlations of $\Lambda-p(\bar{\Lambda}-\bar{p})$ and $\Lambda-\bar{p}(\bar{\Lambda}-p)$ as functions of centrality in $\mathrm{Au}+\mathrm{Au}$ collisions at $200 \mathrm{GeV}$ [62].

is higher than that of mesons, and the correlation strength increases with the average $p_{T}$ of the particle pair in the correlation. Future differential measurements versus the pair average $p_{T}$ and further correlations between identified particles may provide a better insight into the correlation strength between the CME- and CVE-related correlations.

\section{Chiral Magnetic Wave}

The CMW is a signature of the chiral symmetry restoration in the QGP and consists of actually two chiral seamless 


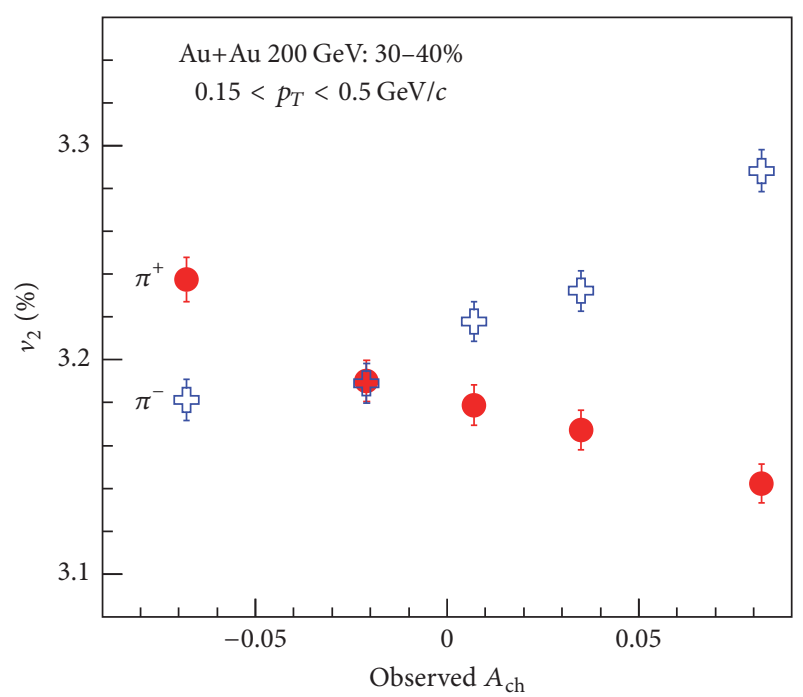

(a)

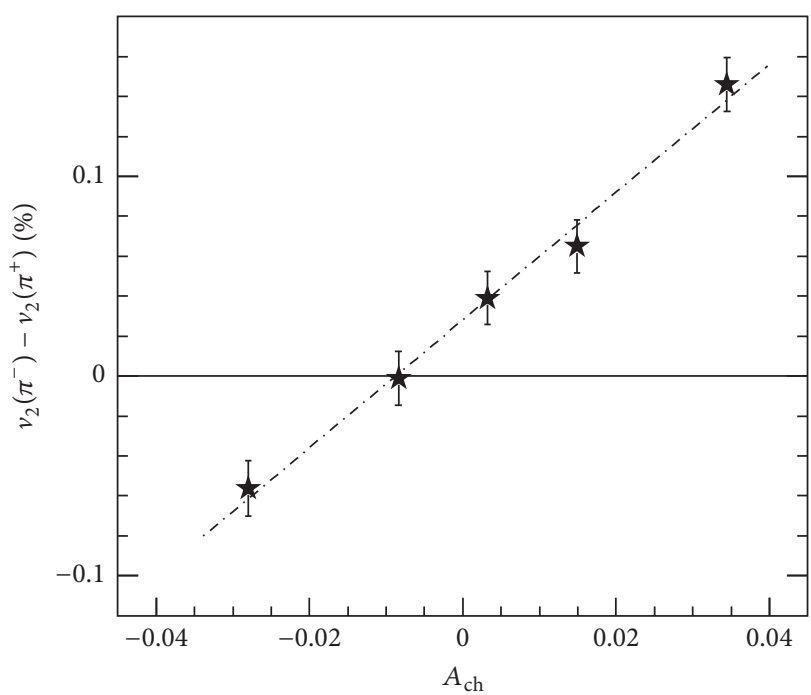

(b)

Figure 14: (a) Pion $v_{2}$ as a function of observed charge asymmetry and (b) $v_{2}$ difference between $\pi^{-}$and $\pi^{+}$as a function of charge asymmetry with the tracking efficiency correction, for $30-40 \% \mathrm{Au}+\mathrm{Au}$ collisions at $200 \mathrm{GeV}$ [80].

modes traveling at the same speed [11]: the right-handed (left-handed) wave transports the right-handed (left-handed) density and current in the direction parallel (antiparallel) to the $\vec{B}$ direction. A more general analysis [84] studied various possible collective modes based on a non-neutralbackground QGP (i.e., with nonzero $\mu$ and/or $\mu_{5}$ ) in external electric and/or magnetic fields and found a new type of collective motion, the chiral electric wave (CEW), arising from CESE and propagating in parallel/antiparallel to the $\vec{E}$ field. In symmetric collisions there should be no net electric field on average, but asymmetric collisions like $\mathrm{Cu}+\mathrm{Au}$ could provide a test ground for the CEW measurements.

5.1. Electric Quadrupole Observable. The CMW will induce a finite electric quadrupole moment of the collision system, with additional positive (negative) charge at the "poles" ("equator") of the produced fireball [11]. This electric quadrupole, if boosted by radial flow, will lead to chargedependent elliptic flow. Let us take pions as an example: on top of the baseline $v_{2}^{\text {base }}\left(\pi^{ \pm}\right)$, the CMW will lead to [11]

$$
v_{2}\left(\pi^{ \pm}\right)=v_{2}^{\text {base }}\left(\pi^{ \pm}\right) \mp\left(\frac{q_{e}}{\bar{\rho}_{e}}\right) A_{\mathrm{ch}}
$$

where $q_{e}, \bar{\rho}_{e}$, and $A_{\mathrm{ch}}=\left(N^{+}-N^{-}\right) /\left(N^{+}+N^{-}\right)$are the quadrupole moment, the net charge density, and the charge asymmetry of the collision event, respectively. $\left\langle A_{\mathrm{ch}}\right\rangle$ is always positive, so the $A_{\mathrm{ch}}$-integrated $v_{2}$ of $\pi^{-}\left(\pi^{+}\right)$should be above (below) the baseline owing to the CMW. However, the baseline $v_{2}$ may be different for $\pi^{+}$and $\pi^{-}$because of several other physics mechanisms $[85,86]$. Therefore, it is less ambiguous to study the CMW via the $A_{\mathrm{ch}}$ dependence of pion $v_{2}$ than via the $A_{\mathrm{ch}}$-integrated $v_{2}$.

Figure 14 [80] takes 30-40\% $200 \mathrm{GeV} \mathrm{Au}+\mathrm{Au}$, for example, and shows pion $v_{2}$ as a function of $A_{\mathrm{ch}}$ in panel (a). $v_{2}\left(\pi^{-}\right)$ increases with $A_{\text {ch }}$, whereas $v_{2}\left(\pi^{+}\right)$decreases with a similar magnitude of the slope. Note that $v_{2}$ was integrated over a narrow low- $p_{T}$ range $\left(0.15<p_{T}<0.5 \mathrm{GeV} / c\right)$ to focus on the soft physics of the CMW. This $p_{T}$ selection also ensures that the $\left\langle p_{T}\right\rangle$ is independent of $A_{\mathrm{ch}}$ and is the same for $\pi^{+}$ and $\pi^{-}$, so that the $v_{2}$ splitting is not a trivial $\left\langle p_{T}\right\rangle$ effect. This $v_{2}$ splitting was also confirmed by ALICE results for $\mathrm{Pb}+\mathrm{Pb}$ collisions at $2.76 \mathrm{TeV}$ [82]. The $v_{2}$ difference between $\pi^{-}$and $\pi^{+}$is fitted with a straight line in panel (b). The slope parameter $r$, or presumably $2 q_{e} / \bar{\rho}_{e}$ from (10), is positive and qualitatively consistent with the expectation of the CMW picture. The fit function is nonzero at $\left\langle A_{\mathrm{ch}}\right\rangle$, indicating the $A_{\mathrm{ch}}$-integrated $v_{2}$ for $\pi^{-}$and $\pi^{+}$are different, which was also observed in [87].

STAR followed the same procedure as shown in Figure 14 to retrieve the slope parameter $r$ as a function of centrality for $\mathrm{Au}+\mathrm{Au}$ collisions at 200,62.4, 39, 27, 19.6, 11.5, and $7.7 \mathrm{GeV}$, as shown in Figure 15 [80]. A similar rise-and-fall trend is observed in the centrality dependence of the slope parameter for all the beam energies except 11.5 and $7.7 \mathrm{GeV}$, where the slopes are consistent with zero with large statistical uncertainties. It was argued [85] that at lower beam energies the $A_{\mathrm{ch}^{-}}$ integrated $v_{2}$ difference between particles and antiparticles can be explained by the effect of quark transport from the projectile nucleons to mid-rapidity, assuming that the coalescence mechanism still holds and that $v_{2}$ of transported quarks is larger than that of produced ones. The same model, however, suggested a negative slope for $v_{2}\left(\pi^{-}\right)-v_{2}\left(\pi^{+}\right)$as a function of $A_{\mathrm{ch}}$ [88], which is contradicted by the data.

As was done in the CME case, contributions from conventional physics were checked via the same analysis of the Monte Carlo events from UrQMD [60]. For $\mathrm{Au}+\mathrm{Au}$ collisions at $200 \mathrm{GeV}$, the slopes extracted from UrQMD events are consistent with zero for the $10-70 \%$ centrality range, where the signal from the real data is prominent. 


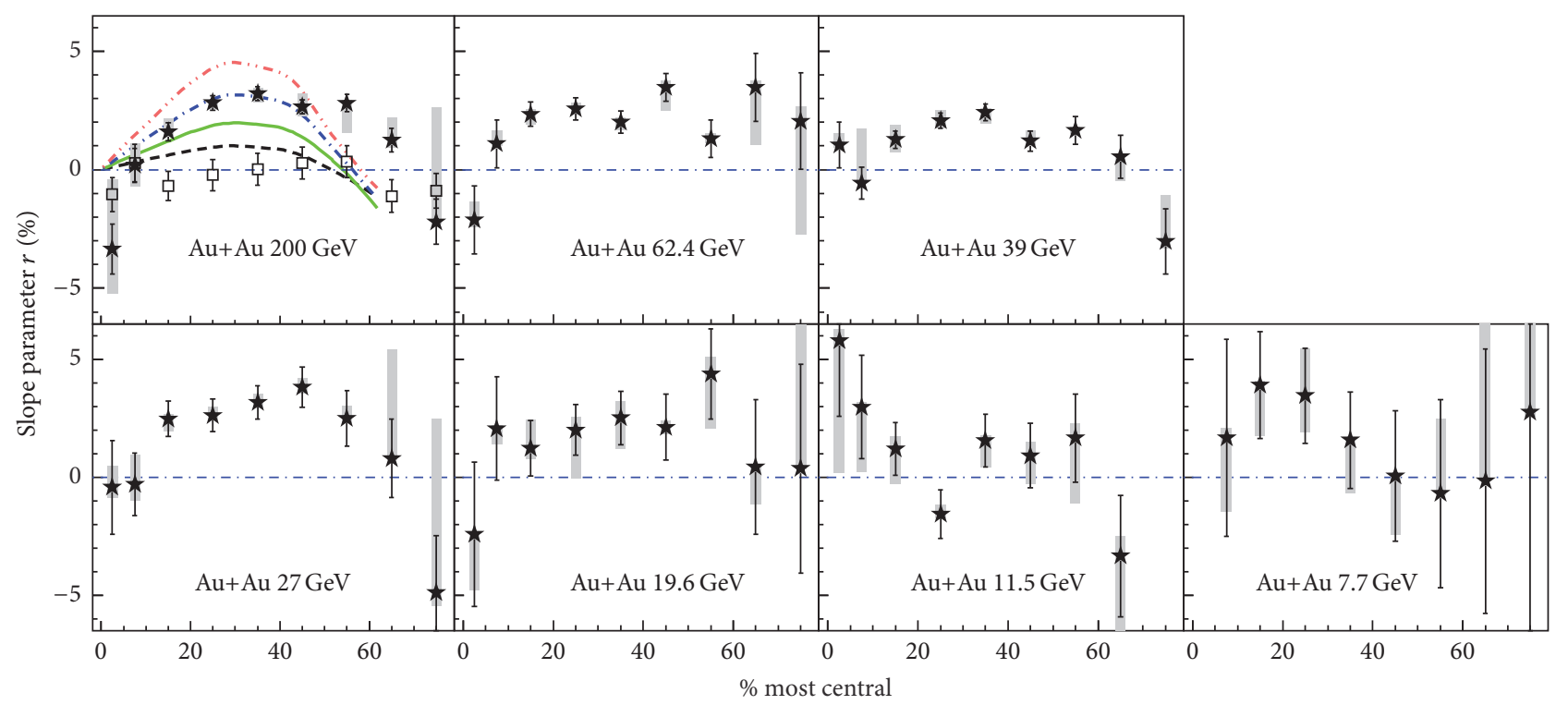

$\square$ UrQMD $\begin{aligned} & \text { CMW } \\ & -\cdots-\tau=6 \mathrm{fm} / c \\ & --\tau=5 \mathrm{fm} / c \\ & -\tau=4 \mathrm{fm} / c \\ & --\tau=3 \mathrm{fm} / c\end{aligned}$

FIGURE 15: The slope parameter $r$ as a function of centrality for $\mathrm{Au}+\mathrm{Au}$ collisions at 7.7-200 GeV [80]. The gray bands represent the systematic errors. For comparison, we also show the UrQMD calculations [60] and the calculations of the CMW [81] with different duration times.

Similarly, the AMPT event generator [70, 71] also yields slopes consistent with zero (not shown here). On the other hand, the simplified CMW calculations [81] demonstrate a centrality dependence of the slope parameter similar to the data. Recently a more realistic implementation of the CMW [89] confirmed that the CMW contribution to $r$ is sizable, and the centrality dependence of $r$ is qualitatively similar to the data. A quantitative comparison between data and theory requires further work on both sides. For example, the STAR experiment only measured $A_{\mathrm{ch}}$ in a pseudorapidity slice $(|\eta|<1)$ of an event, instead of that of the whole collision system. $A_{\text {ch }}$ for these two cases are expected to be proportional to each other, but the determination of the ratio will be model dependent.

Figure 16 shows a comparison in the slope parameter $r$ between STAR results for $200 \mathrm{GeV} \mathrm{Au+Au} \mathrm{[80]} \mathrm{and} \mathrm{ALICE}$ results for $2.76 \mathrm{TeV} \mathrm{Pb}+\mathrm{Pb}$ [82]. Overall, the slopes are surprisingly similar when considering the different collision energies and multiplicities, as well as the different kinematic acceptance: the STAR data estimated $v_{2}$ for charged pions with $0.15<p_{T}<0.5 \mathrm{GeV} / c$ and $|\eta|<1$, while the ALICE data are for unidentified hadrons with $0.2<p_{T}<5 \mathrm{GeV} / \mathrm{c}$ and $|\eta|<0.8$. Note that, in central and peripheral collisions, the STAR slopes at $200 \mathrm{GeV}$ are consistent with zero, while the ALICE slopes at $2.76 \mathrm{TeV}$ still remain positive-definite. This difference leaves room for potential backgrounds in the measurements.

The measurement of $v_{2}\left(A_{\mathrm{ch}}\right)$ requires a correction to the observed $A_{\mathrm{ch}}$ because of the finite detector tracking efficiency. A novel correlator [90] that is less dependent on efficiency was proposed:

$$
\begin{aligned}
\left\langle\left\langle\cos \left[n\left(\varphi_{1}-\varphi_{2}\right)\right] q_{3}\right\rangle\right\rangle & \\
= & \left\langle\cos \left[n\left(\varphi_{1}-\varphi_{2}\right)\right] q_{3}\right\rangle \\
& -\left\langle\cos \left[n\left(\varphi_{1}-\varphi_{2}\right)\right]\right\rangle\left\langle q_{3}\right\rangle .
\end{aligned}
$$

Here $\varphi_{1}$ and $\varphi_{2}$ are the azimuthal angles of particles 1 and 2 , and $q_{3}$ is the charge $( \pm 1)$ of particle 3 . The single brackets represent the average over particles and events, and the double bracket denotes the cumulant. In the absence of charge-dependent correlations, this correlator should be equal to zero. When the charge of the third particle is averaged over all particles in a specified kinematic acceptance of an event, the mean is equal to the charge asymmetry; that is, $\left\langle q_{3}\right\rangle=A_{\text {ch }}$.

The three-particle correlator (as in (11)) for the 2nd harmonic was measured by ALICE and is presented in Figure 17 as a function of centrality in $\mathrm{Pb}+\mathrm{Pb}$ collisions at $2.76 \mathrm{TeV}$ [82]. The correlation strength substantially increases in more peripheral collisions, which can be caused by a combination of several factors. For example, the magnetic field strength increases with the impact parameter and causes the stronger correlations due to the CMW. In addition, the LCC effect could play a role [90], together with other unknown sources of correlation, which is a similar case as the slope parameter discussed above. 


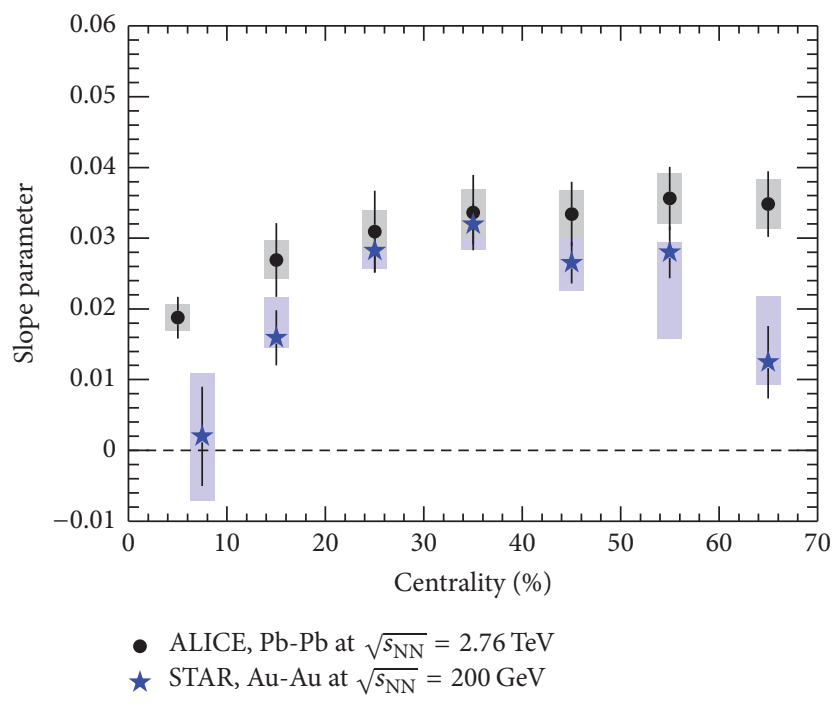

FIGURE 16: Slope parameter $r$ as a function of centrality for $200 \mathrm{GeV}$ $\mathrm{Au}+\mathrm{Au}$ [80] and $2.76 \mathrm{TeV} \mathrm{Pb}+\mathrm{Pb}$ [82]. Statistical (systematic) uncertainties are indicated by vertical bars (shaded boxes).

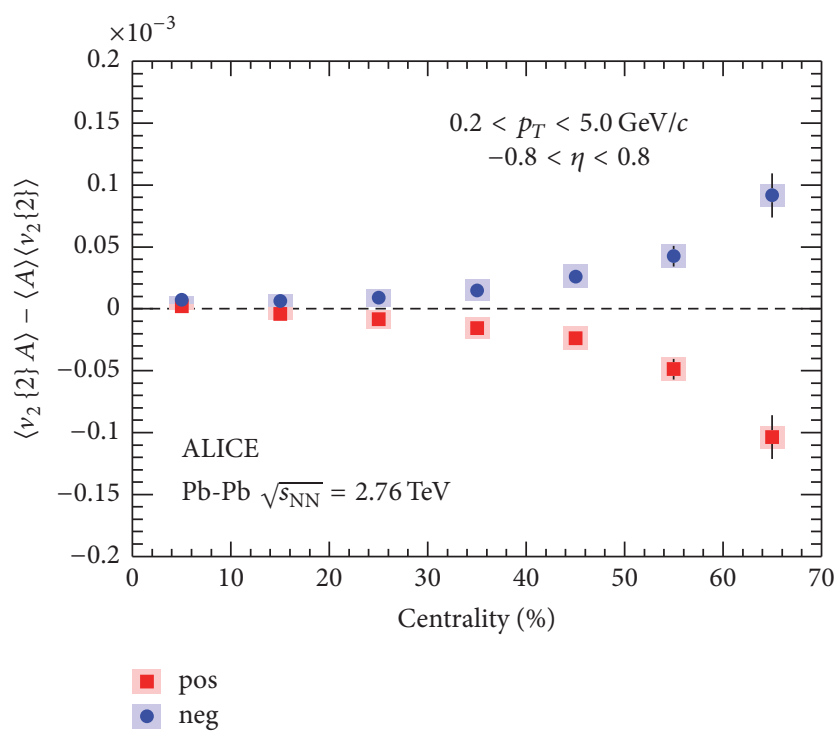

FIGURE 17: Three-particle correlator for the second harmonic, for positive (red squares) and negative (blue circles) particles for $2.76 \mathrm{TeV} \mathrm{Pb}+\mathrm{Pb}$ [82]. Statistical (systematic) uncertainties are indicated by vertical bars (shaded boxes).

5.2. Possible Backgrounds. It was argued [91] that LCC at freeze-out, when convoluted with the characteristic shape of $v_{2}(\eta)$ and $v_{2}\left(p_{T}\right)$, may provide a qualitative explanation for the finite $v_{2}$ slope observed from data. A realistic estimate of the contribution of this mechanism turns out to be smaller than the measurement by an order of magnitude [80]. Reference [91] also proposes a test with the $v_{3}$ measurement, and the corresponding slope parameters for $v_{3}$ were reported by STAR to be consistent with zero [92], which further suggests the smallness of this effect. ALICE measured the three-particle correlator multiplied by $\left\langle d N_{\mathrm{ch}} / d \eta\right\rangle$ for the $3 \mathrm{rd}$ harmonic and the 4th harmonic [82]. In both cases, the centrality dependence of the charge dependence is flat, in contrast to the 2nd harmonic that has a significant centrality dependence. This may indicate a different nature of the correlation or reflect a weaker centrality dependence of $v_{3}$ compared with that of $v_{2}$. Future measurements of these higher harmonics with better precision will shed light on the true origin of this correlator.

Recently a hydrodynamic study [93] claimed that simple viscous transport of charges, combined with certain specific initial conditions, will lead to a sizable $v_{2}$ splitting of charged pions. To have their results of pion splitting resemble data, the authors had to invoke a crucial relation between isospin chemical potential and the electric charge asymmetry, which needs to be verified. Furthermore, certain predictions of this model (e.g., splitting for kaons) appear to be contradicted by experimental data [94]. The validity of such an idea needs to be thoroughly vetted by realistic viscous hydrodynamic simulations. Nevertheless, this study raises an important point: the normal (viscous hydrodynamical) transport of charges should be quantitatively understood to make a firm case for the observation of anomalous charge transport via the CMW.

\section{Future Measurements}

The confirmation of the experimental observation of several chiral anomalous effects will bring forth an exciting program to directly study the nonperturbative sector of QCD. Future experimental measurements should aim at more detailed study of the observed signals as well as understanding the background effects. Previous sections have covered a few such topics: initial magnetic field and vorticity, correlations with identified particles, higher-harmonic correlations, BESII, and U+U collisions. In the following, we will focus on the event shape engineering (ESE) and isobaric collisions.

6.1. Event Shape Engineering. Flow-related backgrounds could be potentially removed via ESE $[75,95]$, with which spherical events or subevents are selected, so that the particles of interest therein carry zero $v_{2}$. A previous attempt was made with the charge separation observable of CMAC (roughly equivalent to $\gamma$ ), as a function of event-by-event "observed $v_{2}$ " [63]. However, there are several issues in this approach that prevent a clear interpretation of the result. Reference [51] studied the flow vector $\vec{q}=\left(q_{x}^{A}, q_{y}^{A}\right)$ of the subevent of interest, $A$ :

$$
\begin{aligned}
& q_{x}^{A}=\frac{1}{\sqrt{N}} \sum_{i}^{N} \cos \left(2 \varphi_{i}^{A}\right), \\
& q_{y}^{A}=\frac{1}{\sqrt{N}} \sum_{i}^{N} \sin \left(2 \varphi_{i}^{A}\right),
\end{aligned}
$$

and found that $q^{2}$ is a good handle on event shape.

Figure 18 shows the event plane resolution $\left(R^{B}\right)$ for the subevents $B 1$ and $B 2$ (a) and the true elliptic flow $v_{2}{ }^{A}$ and the corrected $v_{2}{ }^{\text {observe }}$ as functions of $q^{2}$ (b), from AMPT 


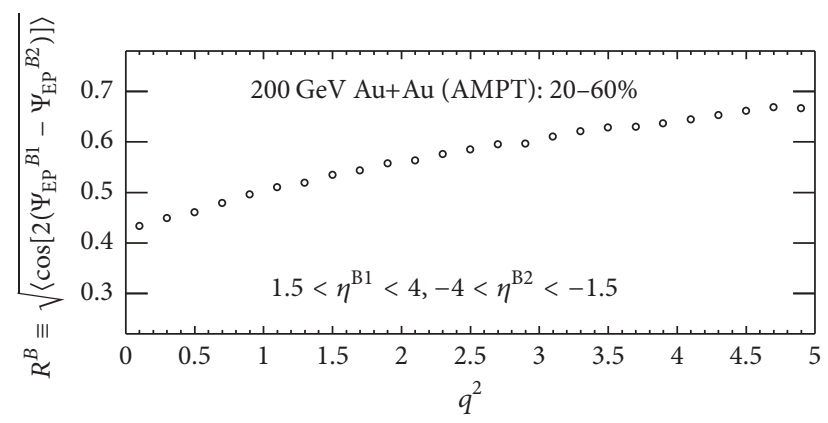

(a)

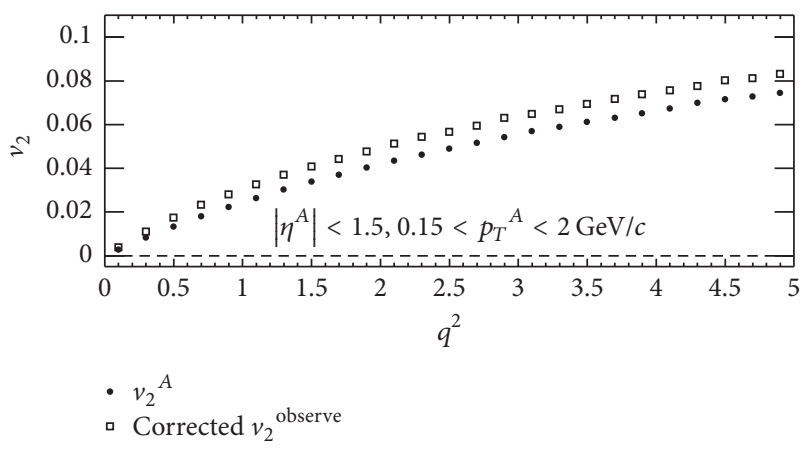

(b)

FIGURE 18: The subevent plane resolution (a), and the true elliptic flow $v_{2}{ }^{\mathrm{A}}$ and the corrected $v_{2}{ }^{\text {observe }}$ as functions of $q^{2}(\mathrm{~b})$, from AMPT simulations [51].

simulations of 20-60\% Au+Au collisions at $\sqrt{s_{\mathrm{NN}}}=200 \mathrm{GeV}$ [51]. Each AMPT event has been divided into three subevents according to pseudorapidity, $\eta$ : subevent $A$ contains particles of interest with $|\eta|<1.5$, and subevent $B 1$ (B2) serves as a subevent plane using particles with $1.5<\eta<4$ $(-4<\eta<-1.5)$. Flow fluctuation causes a positive correlation in flow between subevents in the same event, and as a result, $R^{B}$ for subevent $B 1$ (B2) increases with $q^{2}$ for subevent $A$. Figure 18(b) displays a discrepancy between $v_{2}{ }^{A}$ and the corrected $v_{2}{ }^{\text {observe }}$, owing to the difference between the reaction plane and the participant plane [96], in terms of nonflow and flow fluctuation. What matters more is the fact that both $v_{2}$ values decrease with $q^{2}$ and drop to $(0,0)$, which demonstrates $q$ 's capability of selecting spherical subevents in the second harmonic.

Figure 19(a) presents the $\gamma$ correlators multiplied by the number of participating nucleons, $N_{\text {part }}$, as functions of $q^{2}$, for 20-60\% AMPT events of Au+Au collisions at $200 \mathrm{GeV}$ [51]. For both the same-charge and the opposite-charge correlators, the true $\gamma^{A}$ and the corrected $\gamma^{\text {observe }}$ are consistent with each other within the statistical uncertainties. This indicates that, compared with $v_{2}, \gamma$ is less sensitive to nonflow or flow fluctuation. At larger $q^{2}$, the opposite-charge correlators are above the same-charge correlators, suggesting a finite flow-related background. The opposite- and same-charge correlators converge at small $q^{2}$. Figure 19(b) shows $N_{\text {part }} \Delta \gamma \equiv$ $N_{\text {part }}\left(\gamma_{\text {OS }}-\gamma_{\text {ss }}\right)$ versus $q^{2}$, and again, the two observables seem to coincide. Linear fits to both observables yield small

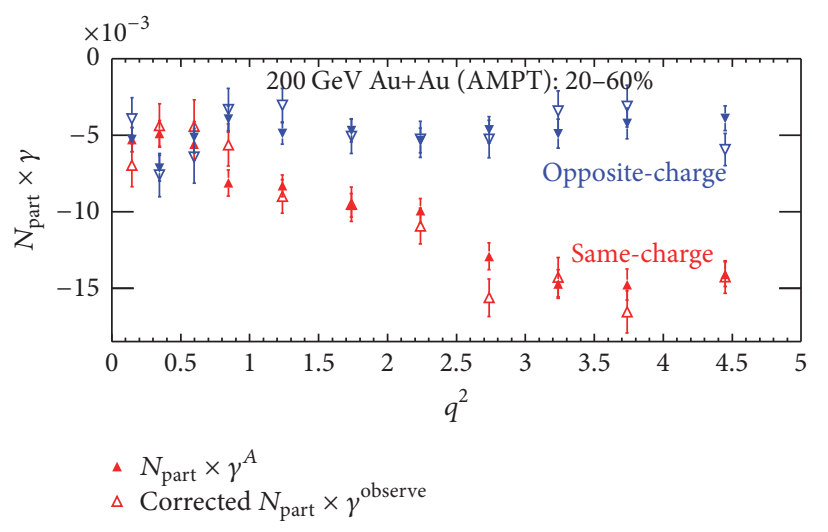

(a)

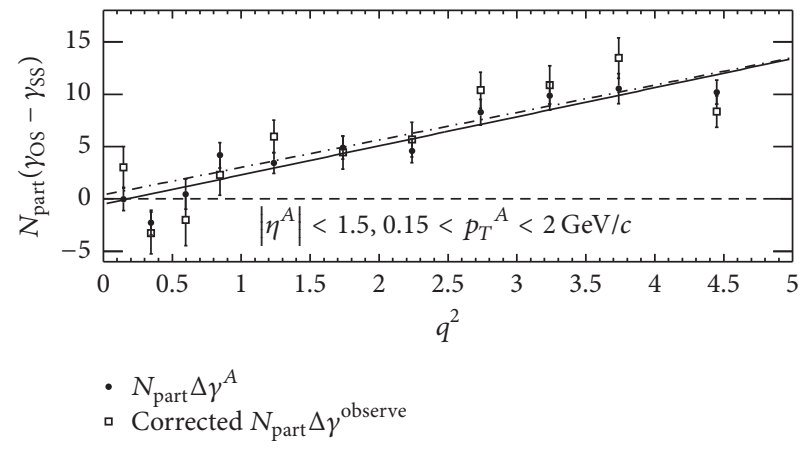

(b)

FIGURE 19: $N_{\text {part }} \times \gamma$ (a) and $N_{\text {part }} \Delta \gamma$ (b) as functions of $q^{2}$, from AMPT simulations [51]. The solid (dashed) line in (b) is a linear fit of the full (open) data points.

intercepts that are consistent with zero. The finite $\Delta \gamma$ values in AMPT events are solely due to background contributions, so the disappearance of background is demonstrated when the "correctable" observable $(\Delta \gamma)$ is projected to zero $q^{2}$. Reference [51] has designed a promising recipe for future measurements to effectively remove flow backgrounds and restore the ensemble average of the CME signal.

6.2. Isobaric Collisions. To disentangle the possible CME signal and the flow-related backgrounds, one can utilize experimental setups to either vary the backgrounds with the signal fixed or vary the signal with the backgrounds fixed. The former approach was carried out by exploiting the prolate shape of the Uranium nuclei [75]. However, it was found that the total multiplicity of detected hadrons is far less dependent on the number of binary collisions than expected [76], so it is very hard to isolate tip-tip collisions (that generate small $v_{2}$ ) from body-body collisions (that generate large $v_{2}$ ). This significantly reduces the lever arm available to manipulate $v_{2}$ in order to separate flow backgrounds from the CME.

The latter approach (with the $v_{2}$-driven backgrounds fixed) can be realized, especially for mid-central or midperipheral events, with collisions of isobaric nuclei, such as ${ }_{96}{ }_{44} \mathrm{Ru}$ and ${ }^{96}{ }_{40} \mathrm{Zr}$ [75]. Ru+Ru and $\mathrm{Zr}+\mathrm{Zr}$ collisions at the same beam energy are almost identical in terms of particle 


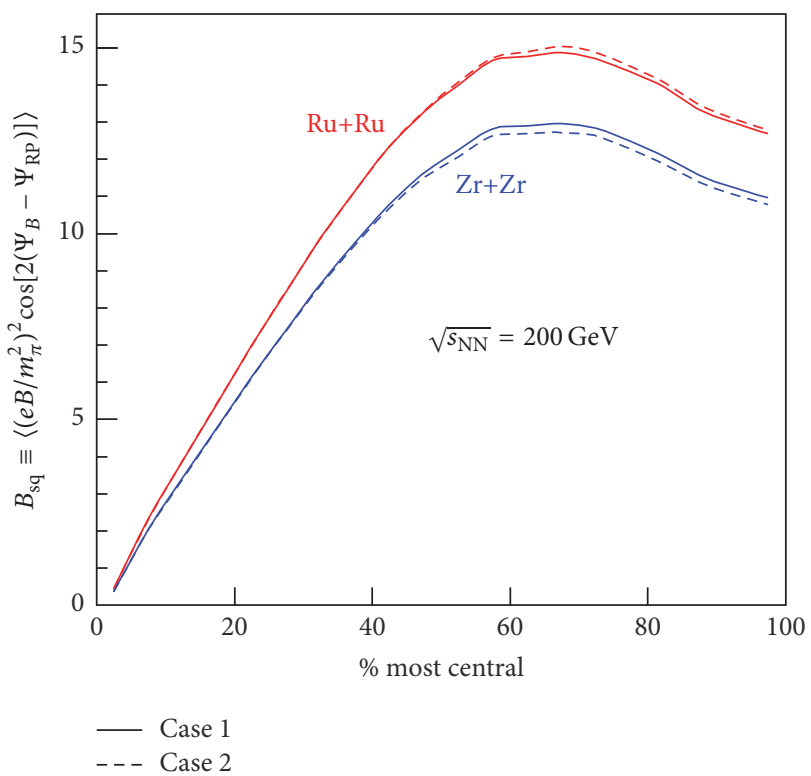

(a)

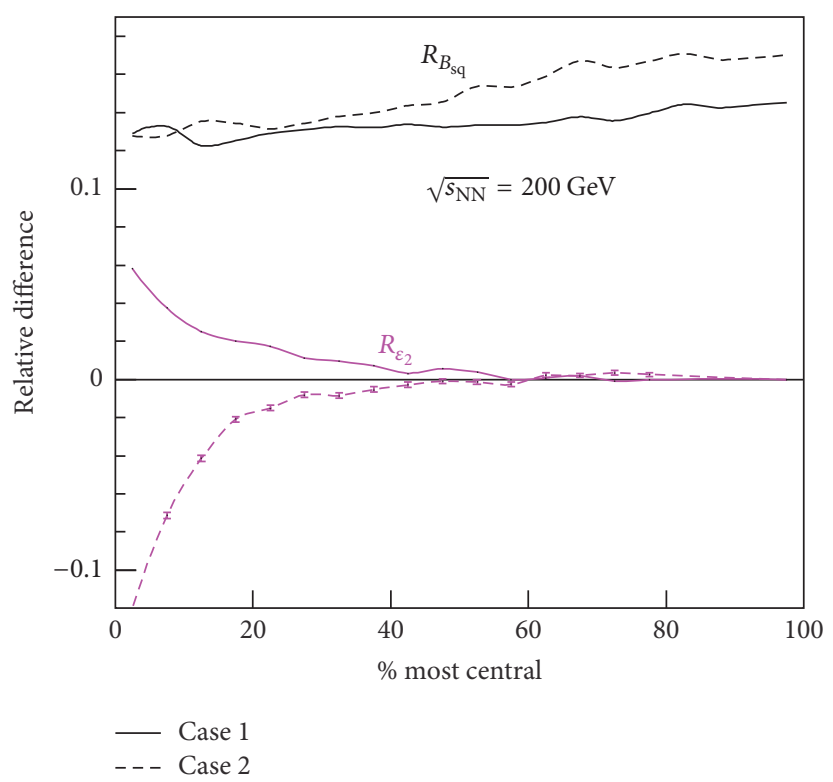

(b)

FIGURE 20: Theoretical calculation [83] of the initial magnetic field squared with correction from azimuthal fluctuation for $\mathrm{Ru}+\mathrm{Ru}$ and $\mathrm{Zr}+\mathrm{Zr}$ collisions at $200 \mathrm{GeV}$ (a) and their relative difference (b) versus centrality. Also shown is the relative difference in initial eccentricity (b). The solid (dashed) lines correspond to the parameter set of case 1 (case 2).

production [83], while the charge difference between $\mathrm{Ru}$ and $\mathrm{Zr}$ nuclei provides a handle on the initial magnetic field. Our current knowledge of the deformity $\left(\beta_{2}\right)$ of $\mathrm{Ru}$ and $\mathrm{Zr}$ is incomplete: $e-A$ scattering experiments (case 1) $[97,98]$ state that $\mathrm{Ru}$ is more deformed $\left(\beta_{2}{ }^{\mathrm{Ru}}=0.158\right)$ than $\mathrm{Zr}\left(\beta_{2}{ }^{\mathrm{Zr}}=\right.$ 0.08 ), while comprehensive model deductions (case 2) [99] tell the opposite that $\beta_{2}{ }^{\mathrm{Ru}}=0.053$ is smaller than $\beta_{2}{ }^{\mathrm{Zr}}=$ 0.217 . This systematic uncertainty has different impacts on the signal (via the initial magnetic field) and the background (via the initial eccentricity) to be discussed later. As a byproduct, $v_{2}$ measurements in central collisions will discern which information source is more reliable regarding the deformity of the $\mathrm{Zr}$ and $\mathrm{Ru}$ nuclei.

Figure 20(a) presents the theoretical calculation [83] of the initial magnetic field squared with correction from azimuthal fluctuation of the magnetic field orientation, $B_{\text {sq }} \equiv$ $\left\langle\left(e B / m_{\pi}^{2}\right)^{2} \cos \left[2\left(\Psi_{B}-\Psi_{\mathrm{RP}}\right)\right]\right\rangle$ (with $m_{\pi}$ being the pion mass and $\Psi_{B}$ the azimuthal angle of the magnetic field), for the two collision systems at $200 \mathrm{GeV}$, using the HIJING model $[19,100] . B_{\mathrm{sq}}$ quantifies the magnetic field's capability of driving the CME signal in the $\gamma$ correlator. For the same centrality bin, the $\mathrm{Ru}+\mathrm{Ru}$ collision produces a significantly stronger magnetic field than $\mathrm{Zr}+\mathrm{Zr}$. Figure 20(b) shows that the relative difference in $B_{\mathrm{sq}}$ between $\mathrm{Ru}+\mathrm{Ru}$ and $\mathrm{Zr}+\mathrm{Zr}$ collisions is approaching $15 \%$ (case 1) or $18 \%$ (case 2) for peripheral events and reduces to about 13\% (both cases) for central events. Figure 20(b) shows the relative difference in the initial eccentricity, $R_{\varepsilon_{2}}$, obtained from the Monte Carlo Glauber simulation. $R_{\varepsilon_{2}}$ is highly consistent with 0 for peripheral events and goes above (below) 0 for the parameter set of case 1 (case 2) in central collisions, because the $\mathrm{Ru}(\mathrm{Zr})$ nucleus is more deformed. The relative difference in $v_{2}$ should closely follow that in eccentricity, so for the centrality range of interest, $20-60 \%$, the $v_{2}$-related backgrounds stay almost the same for $\mathrm{Ru}+\mathrm{Ru}$ and $\mathrm{Zr}+\mathrm{Zr}$ collisions. Reference [83] further carried out the projection for the $\gamma$ measurements in $\mathrm{Ru}+\mathrm{Ru}$ and $\mathrm{Zr}+\mathrm{Zr}$ at $200 \mathrm{GeV}$ (400 million events for each collision type) and concluded that a $5 \sigma$ significance can be achieved for the relative difference in the observable between the two collision systems, assuming the flow backgrounds take up to two-thirds of the observable. The results strongly suggest that the isobaric collisions can serve as an ideal tool to disentangle the signal of the chiral magnetic effect from the $v_{2}$-driven backgrounds. The isobaric collisions may also be used to disentangle the signal of the CMW from background effects.

\section{Summary}

The physics of anomalous transport is an underexplored territory of QCD as a non-Abelian gauge theory. The interplay of quantum anomalies with a strong magnetic field or vorticity enables various novel transport phenomena in chiral systems. In heavy-ion collisions, these phenomena provide a unique probe to the topological properties of the QGP and manifest the charge dependence of the azimuthal distributions of the produced hadrons. In this article, we reviewed the experimental data from RHIC at BNL and the LHC at CERN and provided an evidence for the predicted CME, CVE, and CMW, with magnitudes similar to the theoretical estimates. Although all of these experimental observables contain known conventional backgrounds, no 
compelling alternative explanation at present can describe all of the data without invoking the anomalous chiral effects. Nevertheless, with still miles to go in both experiment and theory to substantiate the existing evidence, we outlined a few such programs that hopefully will be accomplished in the near future.

\section{Competing Interests}

The authors declare that there is no conflict of interests regarding the publication of this paper.

\section{Acknowledgments}

The authors thank Huan Huang and other members of the UCLA Heavy-Ion Physics Group for discussions. This work is supported by a Grant (no. DE-FG02-88ER40424) from US Department of Energy, Office of Nuclear Physics.

\section{References}

[1] D. E. Kharzeev, L. D. McLerran, and H. J. Warringa, “The effects of topological charge change in heavy ion collisions: 'event by event P and CP violation"' Nuclear Physics A, vol. 803, no. 3-4, pp. 227-253, 2008.

[2] D. Kharzeev, "Parity violation in hot QCD: why it can happen, and how to look for it," Physics Letters B, vol. 633, no. 2-3, pp. 260-264, 2006.

[3] D. Kharzeev and A. Zhitnitsky, "Charge separation induced by P-odd bubbles in QCD matter," Nuclear Physics A, vol. 797, no. 1-2, pp. 67-79, 2007.

[4] D. Kharzeev, A. Krasnitz, and R. Venugopalan, "Anomalous chirality fluctuations in the initial stage of heavy ion collisions and parity odd bubbles," Physics Letters, Section B: Nuclear, Elementary Particle and High-Energy Physics, vol. 545, no. 3-4, pp. 298-306, 2002.

[5] I. Iatrakis, S. Lin, and Y. Yin, "Axial current generation by $p$ odd domains in QCD matter," Physical Review Letters, vol. 114, no. 25, Article ID 252301, 2015.

[6] K. Fukushima, D. E. Kharzeev, and H. J. Warringa, "Real-time dynamics of the chiral magnetic effect," Physical Review Letters, vol. 104, no. 21, Article ID 212001, 2010.

[7] A. M. Poskanzer and S. A. Voloshin, "Methods for analyzing anisotropic flow in relativistic nuclear collisions," Physical Review C, vol. 58, no. 3, pp. 1671-1678, 1998.

[8] D. E. Kharzeev and D. T. Son, "Testing the chiral magnetic and chiral vortical effects in heavy ion collisions," Physical Review Letters, vol. 106, no. 6, Article ID 062301, 4 pages, 2011.

[9] D. T. Son and A. R. Zhitnitsky, "Quantum anomalies in dense matter," Physical Review D, vol. 70, no. 7, Article ID 074018, 2004.

[10] M. A. Metlitski and A. R. Zhitnitsky, "Anomalous axion interactions and topological currents in dense matter," Physical Review D, vol. 72, no. 4, Article ID 045011, 2005.

[11] Y. Burnier, D. E. Kharzeev, J. Liao, and H.-U. Yee, "Chiral magnetic wave at finite baryon density and the electric quadrupole moment of the quark-gluon plasma," Physical Review Letters, vol. 107, no. 5, Article ID 052303, 4 pages, 2011.

[12] G. M. Newman, "Anomalous hydrodynamics," Journal of High Energy Physics, vol. 0601, article 158, 2006.
[13] X. G. Huang and J. Liao, "Axial current generation from electric field: chiral electric separation effect," Physical Review Letters, vol. 110, no. 23, Article ID 232302, 2013.

[14] Y. Jiang, X. G. Huang, and J. Liao, "Chiral electric separation effect in the quark-gluon plasma," Physical Review D, vol. 91, no. 4, Article ID 045001, 2015.

[15] Y. Jiang, X. G. Huang, and J. Liao, "Chiral vortical wave and induced flavor charge transport in a rotating quark-gluon plasma," Physical Review D, vol. 92, no. 7, Article ID 071501, 2015.

[16] D. E. Kharzeev, J. Liao, S. A. Voloshin, and G. Wang, "Chiral magnetic and vortical effects in high-energy nuclear collisions-a status report," Progress in Particle and Nuclear Physics, vol. 88, pp. 1-28, 2016.

[17] A. Bzdak and V. Skokov, "Event-by-event fluctuations of magnetic and electric fields in heavy ion collisions," Physics Letters $B$, vol. 710, no. 1, pp. 171-174, 2012.

[18] W. T. Deng and X. G. Huang, "Event-by-event generation of electromagnetic fields in heavy-ion collisions," Physical Review C, vol. 85, no. 4, Article ID 044907, 2012.

[19] W. T. Deng and X. G. Huang, "Electric fields and chiral magnetic effect in Cu + Au collisions," Physics Letters B, vol. 742, pp. 296302, 2015.

[20] J. Bloczynski, X.-G. Huang, X. Zhang, and J. Liao, "Azimuthally fluctuating magnetic field and its impacts on observables in heavy-ion collisions," Physics Letters B, vol. 718, no. 4-5, pp. 1529-1535, 2013.

[21] L. McLerran and V. Skokov, "Comments about the electromagnetic field in heavy-ion collisions," Nuclear Physics A, vol. 929, pp. 184-190, 2014.

[22] X. Guo, S. Shi, N. Xu, Z. Xu, and P. Zhuang, "Magnetic field effect on charmonium production in high energy nuclear collisions," https://arxiv.org/abs/1502.04407.

[23] U. Gürsoy, D. Kharzeev, and K. Rajagopal, "Magnetohydrodynamics, charged currents, and directed flow in heavy ion collisions," Physical Review C, vol. 89, no. 5, Article ID 054905, 2014.

[24] K. Tuchin, "Particle production in strong electromagnetic fields in relativistic heavy-ion collisions," Advances in High Energy Physics, vol. 2013, Article ID 490495, 34 pages, 2013.

[25] Y. Hirono, M. Hongo, and T. Hirano, "Estimation of the electric conductivity of the quark gluon plasma via asymmetric heavyion collisions," Physical Review C, vol. 90, no. 2, Article ID 021903, 2014.

[26] V. Voronyuk, V. D. Toneev, S. A. Voloshin, and W. Cassing, "Charge-dependent directed flow in asymmetric nuclear collisions," Physical Review C, vol. 90, no. 6, Article ID 064903, 2014.

[27] L. Adamczyk, J. K. Adkins, G. Agakishiev et al., "Charge-dependent directed flow in $\mathrm{Cu}+\mathrm{Au}$ collisions at $\sqrt{s_{N N}}=200 \mathrm{GeV}$," https://arxiv.org/abs/1608.04100.

[28] B. I. Abelev, M. M. Aggarwal, Z. Ahammed et al., "System-size independence of directed flow measured at the BNL relativistic heavy-ion collider," Physical Review Letters, vol. 101, no. 25, Article ID 252301, 2008.

[29] Y. Jiang, Z. W. Lin, and J. Liao, "Rotating quark-gluon plasma in relativistic heavy-ion collisions," Physical Review C, vol. 94, no. 4, Article ID 044910, 2016.

[30] M. I. Baznat, K. K. Gudima, A. S. Sorin, and O. V. Teryaev, "Femto-vortex sheets and hyperon polarization in heavy-ion collisions," Physical Review C, vol. 93, no. 3, Article ID 031902, 2016. 
[31] F. Becattini, G. Inghirami, V. Rolando et al., "A study of vorticity formation in high energy nuclear collisions," The European Physical Journal C, vol. 75, article 406, 2015.

[32] F. Becattini, L. Csernai, and D. J. Wang, " $\Lambda$ polarization in peripheral heavy ion collisions," Physical Review C, vol. 88, no. 3, Article ID 034905, 2013.

[33] S. Floerchinger and U. A. Wiedemann, "Fluctuations around Bjorken Flow and the onset of turbulent phenomena," Journal of High Energy Physics, vol. 11, p. 100, 2011.

[34] J. H. Gao, B. Qi, and S. Y. Wang, "Vorticity and magnetic field production in relativistic ideal fluids," Physical Review D, vol. 90, no. 8, Article ID 083001, 2014.

[35] I. Upsal, "Observation of global hyperon polarization in ultrarelativistic heavy ion collisions," Journal of Physics: Conference Series, vol. 736, Article ID 012016, 2016.

[36] STAR Collaboration, STAR Note 0598, https://drupal.star.bnl .gov/STAR/starnotes/public/sn0598.

[37] A. H. Tang and G. Wang, "Procedure for measuring photon and vector meson circular polarization variation with respect to the reaction plane in relativistic heavy-ion collisions," Physical Review C, vol. 94, no. 2, Article ID 024920, 2016.

[38] Y. Hirono, D. E. Kharzeev, and Y. Yin, "Self-similar inverse cascade of magnetic helicity driven by the chiral anomaly," Physical Review D, vol. 92, no. 12, 2015.

[39] A. Ipp, A. Di Piazza, J. Evers, and C. H. Keitel, "Photon polarization as a probe for quark-gluon plasma dynamics," Physics Letters, Section B: Nuclear, Elementary Particle and HighEnergy Physics, vol. 666, no. 4, pp. 315-319, 2008.

[40] K. A. Mamo and H.-U. Yee, "Spin polarized photons and dileptons from axially charged plasma," Physical Review D, vol. 88, no. 11, Article ID 114029, 2013.

[41] K. A. Mamo and H.-U. Yee, "Spin polarized photons from an axially charged plasma at weak coupling: complete leading order," Physical Review D, vol. 93, no. 6, 2016.

[42] Z.-T. Liang and X.-N. Wang, "Globally Polarized Quark-Gluon Plasma in Noncentral A+A Collisions," Physical Review Letters, vol. 94, no. 10, Article ID 102301, 2005.

[43] M. Baznat, K. Gudima, A. Sorin, and O. Teryaev, "Helicity separation in heavy-ion collisions," Physical Review C, vol. 88, no. 6, Article ID 061901, 2013.

[44] W.-T. Deng and X.-G. Huang, "Vorticity in heavy-ion collisions," Physical Review C, vol. 93, no. 6, Article ID 064907, 2016.

[45] L. Adamczyk, J. K. Adkins, G. Agakishiev et al., "Fluctuations of charge separation perpendicular to the event plane and local parity violation in $\sqrt{s_{N N}}=200 \mathrm{GeV} \mathrm{Au}+$ Au collisions at the BNL Relativistic Heavy Ion Collider," Physical Review C, vol. 88, no. 6, Article ID 064911, 2013.

[46] B. I. Abelev, M. M. Aggarwal, Z. Ahammed et al., "Azimuthal charged-particle correlations and possible local strong parity violation," Physical Review Letters, vol. 103, no. 25, Article ID 251601, 2009.

[47] B. I. Abelev, M. M. Aggarwal, Z. Ahammed et al., "Observation of charge-dependent azimuthal correlations and possible local strong parity violation in heavy-ion collisions," Physical Review C, vol. 81, no. 5, Article ID 54908, 2010.

[48] L. Adamczyk, J. K. Adkins, G. Agakishiev et al., "Beam-energy dependence of charge separation along the magnetic field in Au+Au collisions at RHIC," Physical Review Letters, vol. 113, no. 1, Article ID 052302, 2014.

[49] B. I. Abelev, J. Adam, D. Adamová et al., "Charge separation relative to the reaction plane in $\mathrm{Pb}-\mathrm{Pb}$ collisions at $\sqrt{s_{N N}}=$
2.76 TeV,' Physical Review Letters, vol. 110, no. 1, Article ID 012301, 11 pages, 2013.

[50] R. L. Ray and R. S. Longacre, "MEVSIM: a monte carlo event generator for STAR," https://arxiv.org/abs/nucl-ex/0008009.

[51] F. Wen, L. Wen, and G. Wang, "Procedure for removing flow backgrounds from the charge-separation observable perpendicular to the reaction plane in heavy-ion collisions," https:// arxiv.org/abs/1608.03205.

[52] G. Wang, "Search for chiral magnetic effects in high-energy nuclear collisions," Nuclear Physics A, vol. 904-905, pp. 248c255c, 2013.

[53] P. Tribedy, "Charge sensitive cumulants and flow in $U+U$ collisions from STAR," in Proceedings of the Workshop on Chirality, Vorticity and Magnetic Field in Heavy Ion Collisions, 2016, https://drupal.star.bnl.gov/STAR/files/talk_43.pdf.

[54] V. Skokov, P. Sorensen, V. Koch et al., "Chiral magnetic effect task force report," https://arxiv.org/abs/1608.00982.

[55] S. A. Voloshin, "Parity violation in hot QCD: how to detect it," Physical Review C, vol. 70, no. 5, 2004.

[56] A. Bzdak, V. Koch, and J. Liao, "Remarks on possible local parity violation in heavy ion collisions," Physical Review $C$, vol. 81, no. 3, Article ID 031901, 4 pages, 2010.

[57] N. N. Ajitanand, S. Esumi, and R. A. Lacey, "P- and CP-odd ects in hot and dense matter," in Proceedings of the RBRC Workshops, vol. 96, p. 230, 2010.

[58] R. A. Lacey, R. Wei, J. Jia, N. N. Ajitanand, J. M. Alexander, and A. Taranenko, "Initial eccentricity fluctuations and their relation to higher-order flow harmonics," Physical Review C, vol. 83, no. 4, 2011.

[59] N. N. Ajitanand, "Application of a multi-particle correlation method to detect charge asymmetry in $200 \mathrm{GeV} \mathrm{Au}+\mathrm{Au}$ Collisions," in Proceedings of the Workshop on Chirality, Vorticity and Magnetic Field in Heavy Ion Collisions, 2016, https://drupal .star.bnl.gov/STAR/files/Ajit_Parity_Star_chirality_1.pdf.

[60] S. A. Bass, M. Belkacem, M. Bleicher et al., "Microscopic models for ultrarelativistic heavy ion collisions," Progress in Particle and Nuclear Physics, vol. 41, pp. 255-369, 1998.

[61] M. Gyulassy and X.-N. Wang, "HIJING 1.0: a Monte Carlo program for parton and particle production in high energy hadronic and nuclear collisions," Computer Physics Communications, vol. 83, no. 2-3, pp. 307-331, 1994.

[62] F. Zhao, " $\Lambda\left(K_{S}^{0}\right)-h^{ \pm}$and $\Lambda-p$ azimuthal correlations with respect to event plane and search for chiral magnetic and vortical effects," Nuclear Physics A, vol. 931, pp. 746-751, 2014.

[63] L. Adamczyk, J. K. Adkins, G. Agakishiev et al., "Measurement of charge multiplicity asymmetry correlations in high-energy nucleus-nucleus collisions at $\sqrt{s_{N N}}=200 \mathrm{GeV}$," Physical Review C, vol. 89, no. 4, Article ID 044908, 2014.

[64] A. Bzdak, V. Koch, and J. Liao, "Azimuthal correlations from transverse momentum conservation and possible local parity violation," Physical Review C, vol. 83, no. 1, 2011.

[65] S. Pratt, S. Schlichting, and S. Gavin, "Effects of momentum conservation and flow on angular correlations observed in experiments at the BNL Relativistic Heavy Ion Collider," Physical Review C, vol. 84, no. 2, Article ID 024909, 2011.

[66] A. Bzdak, V. Koch, and J. Liao, "Charge-dependent correlations in relativistic heavy ion collisions and the chiral magnetic effect," in Strongly Interacting Matter in Magnetic Fields, vol. 871 of Lecture Notes in Physics, pp. 503-536, Springer, Berlin, Germany, 2013. 
[67] S. Schlichting and S. Pratt, "Charge conservation at energies available at the BNL Relativistic Heavy Ion Collider and contributions to local parity violation observables," Physical Review C, vol. 83, no. 1, Article ID 014913, 2011.

[68] B. Alver, B. B. Back, M. D. Baker et al., "Charged-particle multiplicity and pseudorapidity distributions measured with the PHOBOS detector in $\mathrm{Au}+\mathrm{Au}, \mathrm{Cu}+\mathrm{Cu}, \mathrm{d}+\mathrm{Au}$, and $\mathrm{p}+\mathrm{p}$ collisions at ultrarelativistic energies," Physical Review C, vol. 83, no. 2, Article ID 024913, 2011.

[69] B. B. Back, M. D. Baker, M. Ballintijn et al., "Centrality and pseudorapidity dependence of elliptic flow for charged hadrons in $\mathrm{Au}+\mathrm{Au}$ collisions at $\sqrt{s_{N N}}=200 \mathrm{GeV}$," Physical Review C, vol. 72, no. 5, Article ID 051901, 2005.

[70] B. Zhang, C. M. Ko, B.-A. Li, and Z.-W. Lin, "Multiphase transport model for relativistic nuclear collisions," Physical Review C, vol. 61, no. 6, Article ID 067901, 2000.

[71] Z.-W. Lin, C. M. Ko, B.-A. Li, and B. Zhang, "Multiphase transport model for relativistic heavy ion collisions," Physical Review C, vol. 72, no. 6, Article ID 064901, 2005.

[72] Z. -W. Lin and C. M. Ko, "Partonic effects on the elliptic flow at relativistic heavy ion collisions," Physical Review C, vol. 65, no. 3, Article ID 034904, 2002.

[73] CMS Collaboration, "Observation of charge-dependent azimuthal correlations in $\mathrm{pPb}$ collisions and its implication for the search for the chiral magnetic effect," https://arxiv.org/abs/1610 .00263 .

[74] A. Kuhlman and U. Heinz, "Multiplicity distribution and source deformation in full-overlap U+U collisions," Physical Review C, vol. 72, no. 3, Article ID 037901, 2005.

[75] S. A. Voloshin, "Testing the chiral magnetic effect with central $U+U$ collisions," Physical Review Letters, vol. 105, no. 17, 2010.

[76] L. Adamczyk, J. K. Adkins, G. Agakishiev et al., "Azimuthal Anisotropy in U + U and Au + Au Collisions at RHIC," Physical Review Letters, vol. 115, no. 22, Article ID 222301, 2015.

[77] J. Bloczynski, X.-G. Huang, X. Zhang, and J. Liao, "Chargedependent azimuthal correlations from AuAu to UU collisions," Nuclear Physics A, vol. 939, pp. 85-100, 2015.

[78] S. A. Voloshin, "Collective phenomena in ultra-relativistic nuclear collisions: anisotropic flow and more," Progress in Particle and Nuclear Physics, vol. 67, no. 2, pp. 541-546, 2012.

[79] S. A. Voloshin, "Results on flow from the ALICE Collaboration," Nuclear Physics A, vol. 904-905, 2013.

[80] L. Adamczyk, J. K. Adkins, G. Agakishiev et al., "Observation of charge asymmetry dependence of pion elliptic flow and the possible chiral magnetic wave in heavy-ion collisions," Physical Review Letters, vol. 114, no. 25, Article ID 252302, 2015.

[81] Y. Burnier, D. E. Kharzeev, J. Liao, and H.-U. Yee, "From the chiral magnetic wave to the charge dependence of elliptic flow," https://arxiv.org/abs/1208.2537.

[82] The ALICE Collaboration, "Charge-dependent flow and the search for the chiral magnetic wave in $\mathrm{Pb}-\mathrm{Pb}$ collisions at $\sqrt{s_{N N}}=$ 2.76 TeV,' Physical Review C, vol. 93, no. 4, Article ID 044903, 2016.

[83] W. Deng, X. Huang, G. Ma, and G. Wang, "Testing the chiral magnetic effect with isobaric collisions," Physical Review C, vol. 94, no. 4, Article ID 041901, 2016.

[84] E. V. Gorbar, V. A. Miransky, I. A. Shovkovy, and X. Wang, "Radiative corrections to chiral separation effect in QED," Physical Review D-Particles, Fields, Gravitation and Cosmology, vol. 88, no. 2, Article ID 025025, 2013.
[85] J. C. Dunlop, M. A. Lisa, and P. Sorensen, "Constituent quark scaling violation due to baryon number transport," Physical Review C, vol. 84, no. 4, Article ID 044914, 6 pages, 2011.

[86] J. Xu, L. Chen, C. M. Ko, and Z. Lin, "Effects of hadronic potentials on elliptic flows in relativistic heavy ion collisions," Physical Review C, vol. 85, no. 4, 2012.

[87] L. Adamczyk, J. K. Adkins, G. Agakishiev et al., "Observation of an energy-dependent difference in elliptic flow between particles and antiparticles in relativistic heavy ion collisions," Physical Review Letters, vol. 110, no. 14, Article ID 142301, 2013.

[88] J. M. Campbell and M. A. Lisa, "Can baryon stopping explain the breakdown of constituent quark scaling and proposed signals of chiral magnetic waves at RHIC?" Journal of Physics: Conference Series, vol. 446, Article ID 012014, 2013.

[89] H. Yee and Y. Yin, "Realistic implementation of chiral magnetic wave in heavy ion collisions," Physical Review C, vol. 89, no. 4, 2014.

[90] S. A. Voloshin and R. Belmont, "Measuring and interpreting charge dependent anisotropic flow," Nuclear Physics A, vol. 931, pp. 992-996, 2014.

[91] A. Bzdak and P. Bozek, "Contributions to the event-by-event charge asymmetry dependence for the elliptic flow of $\pi^{+}$and $\pi^{-}$in heavy-ion collisions," Physics Letters B, vol. 726, no. 1-3, pp. 239-243, 2013.

[92] Q.-Y. Shou, "Charge asymmetry dependence of $\pi / K \pi / K$ anisotropic flow in $\mathrm{Au}+\mathrm{AuAu}+\mathrm{Au}$ and $\mathrm{U}+\mathrm{UU}+\mathrm{U}$ collisions at RHIC," Nuclear Physics A, vol. 931, pp. 758-762, 2014.

[93] Y. Hatta, A. Monnai, and B.-W. Xiao, "Elliptic flow difference of charged pions in heavy-ion collisions," Nuclear Physics A, vol. 947, pp. 155-160, 2016.

[94] Q.-Y. Shou, "Charge asymmetry dependence of $\mathrm{K} \mathrm{v}_{2}$ in $\mathrm{Au}+\mathrm{Au}$ collisions at STAR," in Proceedings of the Workshop on Chirality, Vorticity and Magnetic Field in Heavy Ion Collisions, February 2016, http://starmeetings.physics.ucla.edu/sites/ default/files/qiye_shou.pdf.

[95] J. Schukraft, A. Timmins, and S. A. Voloshin, "Ultra-relativistic nuclear collisions: event shape engineering," Physics Letters B, vol. 719, no. 4-5, pp. 394-398, 2013.

[96] J.-Y. Ollitrault, A. M. Poskanzer, and S. A. Voloshin, "Effect of flow fluctuations and nonflow on elliptic flow methods," Physical Review C, vol. 80, no. 1, Article ID 014904, 2009.

[97] S. Raman, C. W. Nestor Jr., and P. Tikkanen, “Transition probability from the ground to the first-excited $2^{+}$state of eveneven nuclides," Atomic Data and Nuclear Data Tables, vol. 78, no. 1, pp. 1-128, 2001.

[98] B. Pritychenko, M. Birch, B. Singh, and M. Horoi, “Tables of E2 transition probabilities from the first $2^{+}$states in even-even nuclei," Atomic Data and Nuclear Data Tables, vol. 107, pp. 1-139, 2016.

[99] P. Möller, J. R. Nix, W. D. Myers, and W. J. Swiatecki, "Nuclear ground-state masses and deformations," Atomic Data and Nuclear Data Tables, vol. 59, no. 2, pp. 185-381, 1995.

[100] W. Deng and X. Huang, "Event-by-event generation of electromagnetic fields in heavy-ion collisions," Physical Review C, vol. 85, no. 4, Article ID 044907, 2012. 

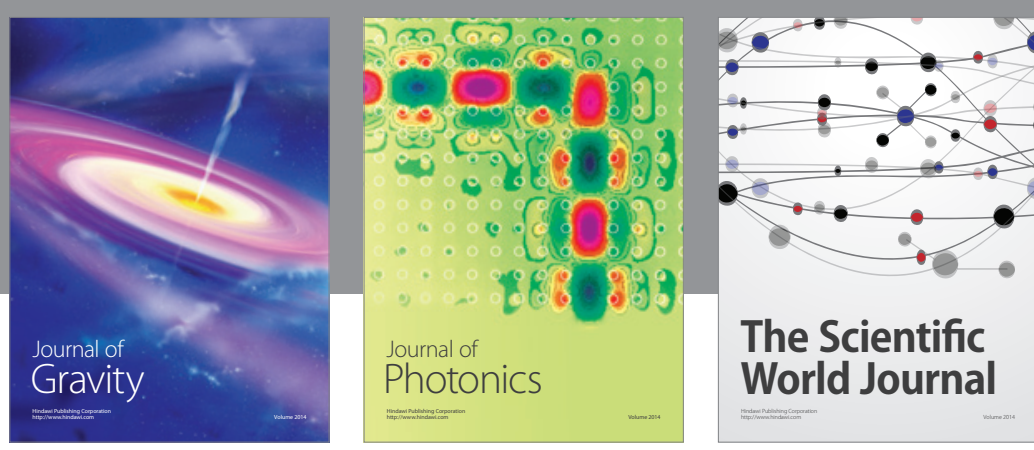

The Scientific World Journal
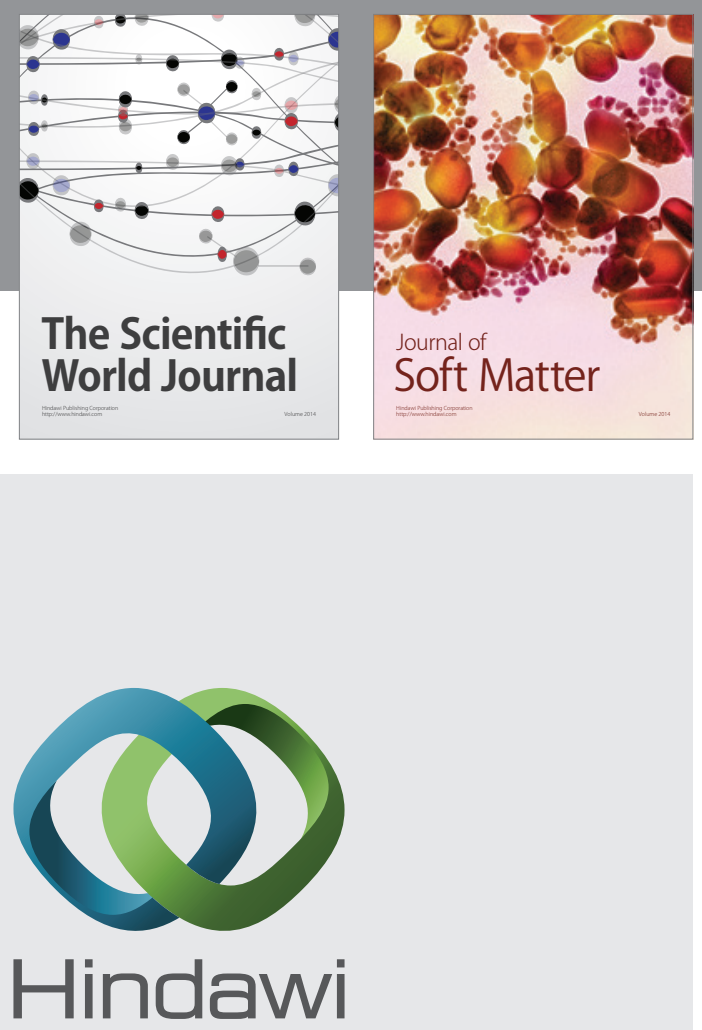

Submit your manuscripts at

https://www.hindawi.com
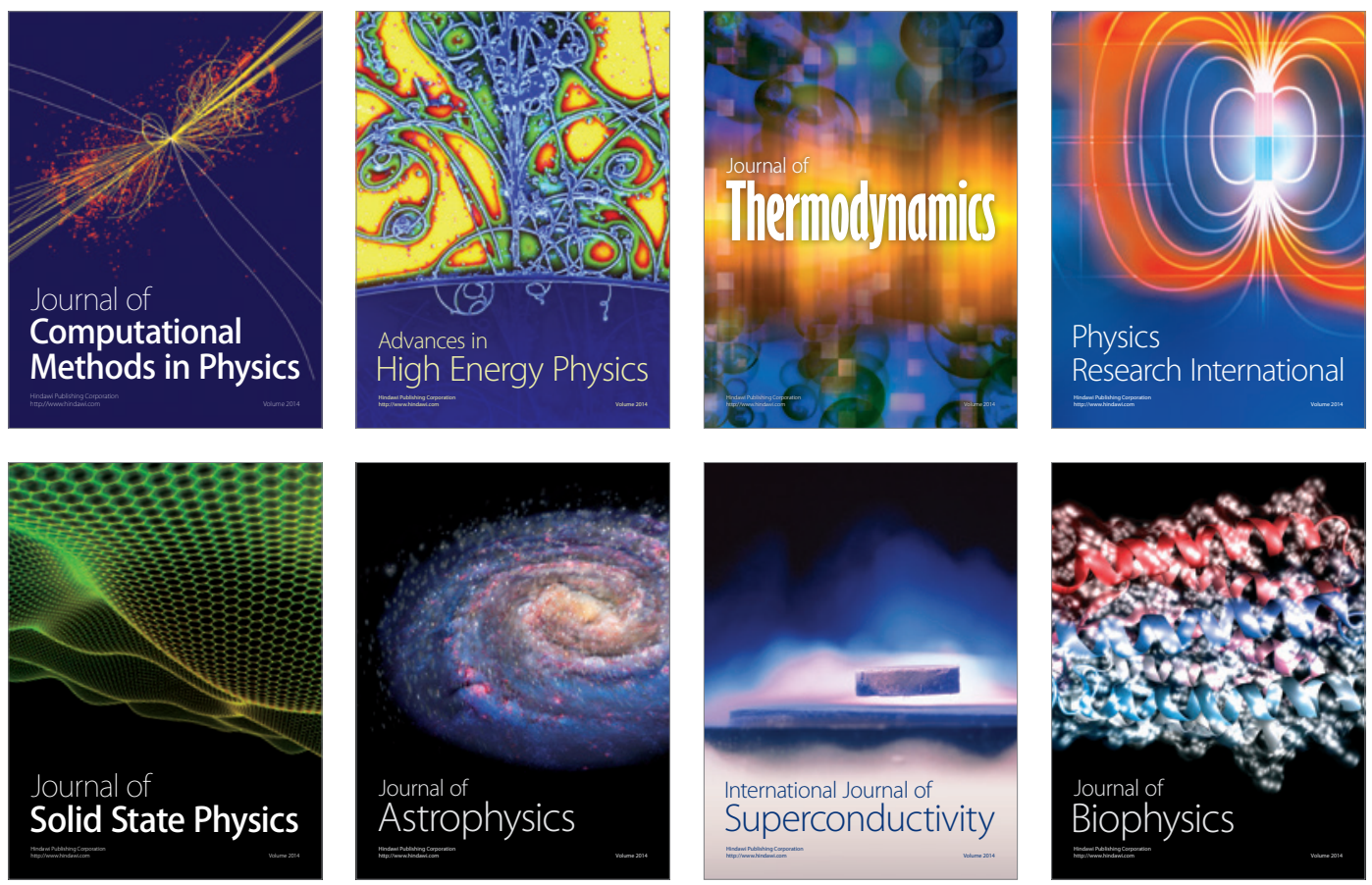
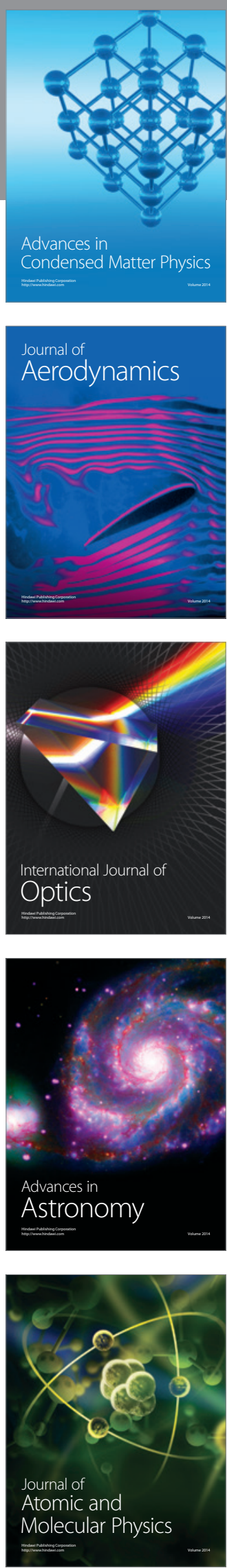